Sharif University of Technology
Scientia Iranica
Transactions E: Industrial Engineering
hCIENTIA

\title{
Medical tourism destinations prioritization using group decision making method with neutrosophic fuzzy preference relations
}

\author{
Y. Yang ${ }^{\mathrm{a}}, \mathrm{J} . \mathrm{Hu}^{\mathrm{a}, *}$, R. Sun ${ }^{\mathrm{a}}$, and X. Chen ${ }^{\mathrm{a}, \mathrm{b}}$ \\ a. School of Business, Central South University, Changsha, China. \\ b. School of Accounting, Hunan University of Commerce, Changsha, China. \\ Received 15 March 2017; received in revised form 4 July 2017; accepted 14 October 2017
}

\author{
KEYWORDS \\ Neutrosophic fuzzy \\ preference relations; \\ Consistency; \\ Aggregation \\ operators; \\ Multiple criteria group \\ decision making; \\ Medical tourism.
}

\begin{abstract}
Medical tourism has developed rapidly worldwide, especially in Asia, and one of the most important problems facing the patient-tourists is the selection of the optimum destination. In this paper, we present a novel Multiple Criteria Group Decision Making (MCGDM) methodology to evaluate and rank the medical tourism destinations based on vague information. A systematic assessment and selection model was constructed by investigating MCGDM problems with Neutrosophic Fuzzy Preference Relations (NFPRs). We began by defining NFPRs, which allowed the patient-tourists lacking information, time, or patience to express their uncertainty and hesitancy about the given preference values. The additive consistency and acceptable consistency for NFPRs were then proposed. Furthermore, the approach to improve consistency of NFPRs was validated and a series of aggregation operators were developed. In addition, we presented a systematic MCGDM method using NFPRs (MCGDM-NFPRs) to rank the medical tourism destinations. Then, our proposed approach was applied to two cases considering different kinds of original data to prioritize medical tourism places. Finally, the applicability and feasibility of the proposed approach were verified by comparison with other previous methods, along with some analyses and a comprehensive discussion.
\end{abstract}

(C) 2018 Sharif University of Technology. All rights reserved.

\section{Introduction}

Medical tourism is a global industry that focuses on obtaining medical treatments in foreign destinations. Factors that have contributed to the rapid development of medical tourism include expensive health care in home country, long waiting lists for certain procedures, the increase in the affordability of overseas traveling,

\footnotetext{
*. Corresponding author. Tel.: +86 13974872488;

Fax: +867318710006

E-mail addresses: gabbylovie@csu.edu.cn (Y. Yang);

hujunhua@csu.edu.cn (J.Hu); sunruixiao@csu.edu.cn (R.

Sun); cxh_csu@csu.edu.cn (X. Chen)
}

doi: $10.24200 /$ sci.2017.4514 the melioration of technology for health treatments in many states, and profitable change rates in destination countries, among others [1]. Traditionally, people preferred to travel from the underdeveloped countries to the more developed ones to avail the advanced medical treatments that were lacking in their homeland $[2,3]$. But, in recent years, this trend has reversed with more people from the developed countries travelling to some third-world countries. Several reasons have contributed to this phenomenon, such as affordable healthcare, easy availability of skilled manpower, rapid development of medical facilities in recent years, fewer restrictions on policies and laws, and so on.

The most popular international healthcare destinations that have also attracted the attention of many 
researchers include South Africa [4], Thailand [5,6], Mongolia [7], Hong Kong [8], Barbado [9], India [10], and South Korea [11]. Medical tourism industry, especially of India, which is researched in this paper, is a rapidly growing sector in the tourism industry and is estimated to have been worth $\$ 3$ billion in 2015 . Furthermore, it is projected to reach $\$ 7-8$ billion by 2020 [12].

Although many studies have been conducted on the social impact of medical tourism [7-9,11,13], there are not many tools available for patients to evaluate the various medical tourism destinations and select the most suitable ones for their needs. Roy et al. [14] argued that this issue could be solved by considering the interests of certain stakeholders, like medical infrastructure, logistics enterprises, and government regulations, in assessing the weight of a multiple criteria set. Eissler et al. [15] developed the understanding of the experience in selecting health care internationally from the perspective of patients. Taken together, the evaluation and selection of medical sites could be regarded as a Multiple Criteria Decision Making (MCDM) problem.

However, due to the great complexity of the real world, it is difficult to obtain enough information about each destination (alternative) under different criteria. Therefore, it is worth considering a pairwise comparison of a set of available alternatives. In these cases, the Preference Relations (PRs) [16], which are a useful tool in modeling decision processes, are always efficiently used to describe opinions of the experts. In recent years, PRs have attracted much attention and undergone developments as the most common representative structures of information in the field of GDM. Due to the uncertainty of decision related problems, it is difficult for a decision maker (DM) to offer a crisp preference degree of pairwise judgments. In order to overcome such issues, the Fuzzy Preference Relations (FPRs) [17,18] and some extensions [19,20] have been proposed.

As one of the most useful extensions of FPRs, the Intuitionistic Preference Relation (IPR) [21] and its extensions have drawn much interest [22-25], which allow DMs to express their affirmation, negation, and hesitation. For example, Chiclana et al. [26] gave the method to tackle a situation with unknown values in reciprocal IPRs by using asymmetric FPR. Based on the above method, Ureña et al. [27] proposed a confidence-consistency driven GDM method for incomplete reciprocal IPRs, which could overcome the computational complexity. In addition, Xu [28] developed a consensus reaching method in GDM according to the compatibility measures. Wang et al. [29] presented an acceptable consistency-based procedure for GDM with IPRs and Wu [30] put forward a Multiple Criteria Group Decision Making (MCGDM) framework with the consistency of IPRs based on the exponential score function. Zeng et al. [31] constructed a novel model for interactive GDM with IPRs. Zhang et al. [32] further investigated the prioritization and aggregation of the IPRs. Furthermore, many extensions of IPRs, such as interval-valued IPRs [33], intuitionistic multiplicative PRs [34], and hesitant-IPRs [35], have been proposed and developed.

The consistency of PRs, which has a direct effect on reaching consistent and reasonable conclusions, is a vital factor in designing good decision making models and has therefore been researched extensively [3639]. Studying consistency is related to the concept of transitivity, such as the max-min transitivity [40,41], the max-max transitivity, the additive transitivity, and so on $[42,43]$. In particular, the additive transitivity, which will be used in this paper, is stronger than restricted max-max and restricted max-min ones and weaker than max-max and max-min ones. Furthermore, many consistency measurements for different kinds of PRs have been studied. Liao et al. [44] introduced multiplicative consistency, perfect multiplicative consistency, and acceptable multiplicative consistency for HFPRs. Wang and Xu [45] developed additive consistency measure and weak consistency measure for the extended hesitant fuzzy linguistic preference relations. Rallabandi et al. [46] proposed an improved consistency ratio for the pairwise comparison matrix. In addition, the aggregation approaches are important to solve MCGDM problems, which could aggregate several values. Many researches have contributed to the aggregation of FPRs; for instance, $\mathrm{Li}$ et al. [47] came up with the conversion of interval multiplicative weights to acceptable interval multiplicative PRs and established an interval multiplicative weight derivation model. Wang and Lin [48] explored the priority weight elicitation for triangular fuzzy multiplicative PRs. Wang [49] presented a linear goal programming framework in order to obtain normalized interval weights from interval FPRs.

We can conclude from all these studies that the exiting forms of FPRs cannot deal with a situation in which experts are hesitant about their judgments due to the lack of information and the complexity of the real environment. It is therefore obvious that consistency is a vital and useful tool to ensure the logic and efficiency of a preference. The aim of this paper is to propose neutrosophic fuzzy preference relations (NFPRs), which permit a DM to express their membership or non-membership regarding the preference of one destination (alternative) over another one and their opinion of the unsure degree of the two values, simultaneously, on the one hand, and to give a novel MCGDM method using NFPRs (MCGDMNPFRs) on the basis of consistency measurements in 
order to evaluate and prioritize the medical tourism destinations, on the other hand.

The remainder of this paper is organized as follows. Section 2 reviews some basic concepts of Neutrosophic Set (NS), Single Valued Neutrosophic Set (SVNS), and the corresponding operational laws. Section 3 introduces the concepts of NFPR, its additive consistency and acceptable consistency according to FPR, IPR and the additive consistency of IPR, and some operations of Single Valued Neutrosophic Element (SVNE). This is followed by Section 4 that describes a method to improve the consistency of NFPRs and proves some properties. The MCGDM-NFPRs model is proposed in Section 5 and evaluated further for medical tourism sites selection in Section 6 by comparing two cases with two previous methods. The conclusion of this study is finally pointed out in Section 7 .

\section{Preliminaries}

In this section, we discuss some definitions, operations, and properties of SVNS as defined in previous studies [50,51], along with the definition of FPR and its additive consistency, which will be used in the rest of the paper.

Definition 2.1 [51]. Let $X$ be a space of points (objects) with generic elements in $X$ denoted by $x$. An SVNS $A$ in $X$ is characterized by truth-membership function $T_{A}(x)$, indeterminacy-membership function $I_{A}(x)$, and falsity-membership function $F_{A}(x)$ for each point $x$ in $X, T_{A}(x), I_{A}(x), F_{A}(x) \in[0,1]$.

For convenience, an SVNS $A=\left\{x, T_{A}(x), I_{A}(x)\right.$, $\left.F_{A}(x) \mid x \in X\right\}$ is denoted by the simplified symbol $A=<T_{A}(x), I_{A}(x), F_{A}(x)>$ for any $x$ in $X$. In this paper, we call $\tilde{a}=\left\langle T_{a}(x), I_{a}(x), F_{a}(x)\right\rangle$ an SVNE, where $x \in X$.

Definition 2.2 [51]. The complement to an SVNS $A=\left\langle T_{A}(x), I_{A}(x), F_{A}(x)\right\rangle$ is denoted by $A^{c}$ and $A^{c}=$ $\left\langle F_{A}(x), 1-I_{A}(x), T_{A}(x)\right\rangle$. The following expressions are defined for the two SVNSs $A$ and $B$ :

1. $A \subseteq B$ if and only if $T_{A}(x) \leq T_{B}(x), I_{A}(x) \leq$ $I_{B}(x), F_{A}(x) \leq F_{B}(x)$ for any $x$ in $X$,

2. $A=B$ if and only if $A \subseteq B$ and $B \subseteq A$.

Definition 2.3 [51]. For two SVNSs $A$ and $B$, the operational relations are defined as follows:

1. $A \cup B=\left\langle\max \left(T_{A}(x), T_{B}(x)\right)\right.$, $\left.\min \left(I_{A}(x), I_{B}(x)\right), \min \left(F_{A}(x), F_{B}(x)\right)\right\rangle$, for any $x$ in $X$,

2. $A \cap B=\left\langle\min \left(T_{A}(x), T_{B}(x)\right)\right.$, $\left.\max \left(I_{A}(x), I_{B}(x)\right), \max \left(F_{A}(x), F_{B}(x)\right)\right\rangle$, for any $x$ in $X$,
3. $A \times B=\left\langle T_{A}(x)+T_{B}(x)-T_{A}(x) T_{B}(x)\right.$,

for any $x$ in $X$.

$$
\left.I_{A}(x) I_{B}(x), F_{A}(x) F_{B}(x)\right\rangle \text {, }
$$

\section{NFPR and its consistency measurements}

This section defines the notion of NFPR based on the definitions of FPR [52] and IPR [53]. The additive consistency and acceptable consistency of NFPR, which are important to ensure the consistent logic of DMs, are proposed based on the additive consistency of IPR [54].

Definition 3.1 [52]. An FPR $P$ on a set of alternatives $X$ on the product set $X \times X$ is a fuzzy set, which is characterized by a membership function $\mu_{P}$, where $\mu_{P}: X \times X \rightarrow[0,1]$.

When the cardinality of $X$ is small, the PR could be represented by a matrix $P=\left(p_{i j}\right)_{n \times n}$, where $p_{i j}$ satisfies the following characteristics:

$$
\begin{aligned}
& 0 \leq p_{i j} \leq 1, \quad p_{i j}+p_{j i}=1, \quad p_{i i}=0.5, \\
& \text { for any } i, j \in\{1,2, \ldots, n\}
\end{aligned}
$$

Definition 3.2 [53]. Let $X=\left\{x_{1}, x_{2}, \ldots, x_{n}\right\}$ be a fixed set, then an IPR $\tilde{R}$ on the set $X$ is represented by a matrix $\tilde{R}=\left(\tilde{r}_{i j}\right)_{n \times n} \subset X \times X$, where $\tilde{r}_{i j}=\left\langle\left(x_{i}, x_{j}\right), \mu\left(x_{i}, x_{j}\right), v\left(x_{i}, x_{j}\right)\right\rangle$ for any $i, j \in$ $\{1,2, \ldots, n\}$. For convenience, $\tilde{r}_{i j}$ is denoted by the simplified symbol, $\tilde{r}_{i j}=\left(\mu_{i j}, v_{i j}\right)$, composed by $\mu_{i j}$ in which $x_{i}$ is preferred to $x_{j}$ and $v_{i j}$ in which $x_{i}$ is nonpreferred to $x_{j}$. Furthermore, $\tilde{r}_{i j}=\left(\mu_{i j}, v_{i j}\right)$ satisfies the following conditions:

$$
\begin{aligned}
& 0 \leq \mu_{i j}+v_{i j} \leq 1 \\
& \mu_{j i}=v_{i j}, \quad v_{j i}=\mu_{i j} \quad \mu_{i i}=v_{i i}=0.5
\end{aligned}
$$$$
\text { for any } i, j \in\{1,2, \ldots, n\} \text {. }
$$

Definition 3.3 [54]. Let $\tilde{R}=\left(\tilde{r}_{i j}\right)_{n \times n}$ be an IPR where $\tilde{r}_{i j}=\left(\mu_{i j}, v_{i j}\right) ; \tilde{R}$ has additive consistency if it satisfies the following conditions:

$$
\begin{aligned}
& \mu_{i j}+\mu_{j k}+\mu_{k i}=\mu_{k j}+\mu_{j i}+\mu_{i k}, \\
& \text { for any } i, j \in\{1,2, \ldots, n\} .
\end{aligned}
$$

According to Eqs. (2) and (3), if $\tilde{R}=\left(\tilde{r}_{i j}\right)_{n \times n}$ is additive consistent, then:

$$
\begin{aligned}
& v_{i j}+v_{j k}+v_{k i}=v_{k j}+v_{j i}+v_{i k}, \\
& \text { for any } i, j \in\{1,2, \ldots, n\} .
\end{aligned}
$$

Definition 3.4 [55]. Given an SVNE $\tilde{a}=\left\langle T_{a}(x)\right.$, $\left.I_{a}(x), F_{a}(x)\right\rangle$, then $S(\tilde{a})=\left(T_{a}(x)-F_{a}(x)\right)\left(1-I_{a}\right.$ 
$(x))$ is called the score function of $\tilde{a}$, and $S^{\prime}(\tilde{a})=$ $T_{a}(x)-F_{a}(x)$ and $H^{\prime}(\tilde{a})=T_{a}(x)+F_{a}(x)$ are the sub-score function and sub-accuracy function, respectively. These three functions can be used as the basis to compare two SVNEs. By taking a prioritized sequence of score function, sub-score function, and sub-accuracy function, an approach is devised for comparing two SVNEs $\tilde{a}=\left\langle T_{a}(x), I_{a}(x), F_{a}(x)\right\rangle$ and $\tilde{b}=\left\langle T_{b}(x), I_{b}(x), F_{b}(x)\right\rangle$ as follows:

- If $S(\tilde{a})>S(\tilde{b})$, then $\tilde{a}$ is greater than $\tilde{b}$, denoted by $\tilde{a}>\tilde{b}$;

- If $S(\tilde{a})>S(\tilde{b})$, then $\tilde{a}$ is smaller than $\tilde{b}$, denoted by $\tilde{a}>\tilde{b}$;

- If $S(\tilde{a})=S(\tilde{b})$, then:

- If $S^{\prime}(\tilde{a})>S^{\prime}(\tilde{b})$, then $\tilde{a}$ is greater than $\tilde{b}$, denoted by $\tilde{a}>\tilde{b}$;

- If $S^{\prime}(\tilde{a})<S^{\prime}(\tilde{b})$, then $\tilde{a}$ is smaller than $\tilde{b}$, denoted by $\tilde{a}>\tilde{b}$;

- If $S^{\prime}(\tilde{a})=S^{\prime}(\tilde{b})$, then:

(a) If $H^{\prime}(\tilde{a})>H^{\prime}(\tilde{b})$, then $\tilde{a}$ is greater than $\tilde{b}$, denoted by $\tilde{a}>\tilde{b}$;

(b) If $H^{\prime}(\tilde{a})<H^{\prime}(\tilde{b})$, then $\tilde{a}$ is smaller than $\tilde{b}$, denoted by $\tilde{a}<\tilde{b}$;

(c) If $H^{\prime}(\tilde{a})=H^{\prime}(\tilde{b})$, then $\tilde{a}$ and $\tilde{b}$ represent the same information, denoted by $\tilde{a}=\tilde{b}$.

Theorem 3.1. Let $\tilde{a}=\left\langle T_{a}(x), I_{a}(x), F_{a}(x)\right\rangle$ be an SVNE, then the score function $S(\tilde{a})=\left(T_{a}(x)-\right.$ $\left.F_{a}(x)\right)\left(1-I_{a}(x)\right)$, the sub-score function $S^{\prime}(\tilde{a})=$ $T_{a}(x)-F_{a}(x)$, and the sub-accuracy function $H^{\prime}(\tilde{a})=$ $T_{a}(x)+F_{a}(x)$ should satisfy the following properties:

1) $-1 \leq S(\tilde{a}) \leq 1$;

2) $-1 \leq S^{\prime}(\tilde{a}) \leq 1$;

3) $0 \leq H^{\prime}(\tilde{a}) \leq 1$.

Definition 3.5. An NFPR $\tilde{A}$ on a fixed set $X=$ $\left\{x_{1}, x_{2}, \ldots, x_{n}\right\}$ is represented by a matrix:

$$
\tilde{A}=\left(\tilde{a}_{i j}\right)_{n \times n} \subset X \times X,
$$

where:

$$
\tilde{a}_{i j}=\left\langle T_{\tilde{a}_{i j}}\left(x_{i j}\right), I_{\tilde{a}_{i j}}\left(x_{i j}\right), F_{\tilde{a}_{i j}}\left(x_{i j}\right)\right\rangle,
$$

for any $i, j \in\{1,2, \ldots, n\}$. For convenience, we let:

$$
\tilde{a}_{i j}=\left\langle T_{i j}, I_{i j}, F_{i j}\right\rangle \text {, }
$$

for any $i, j \in\{1,2, \ldots, n\}$, where $\tilde{a}_{i j}$ is an SVNE composed by the true degree $T_{i j}$, the indeterministic degree $I_{i j}$, and the false degree $F_{i j}$ of $x_{i}$ preferred to $x_{j}$, respectively. Also, $\tilde{a}_{i j}=\left\langle T_{i j}, I_{i j}, F_{i j}\right\rangle$ should also satisfy the following characteristics:

$$
\begin{aligned}
& T_{i j}=F_{j i}, \quad F_{i j}=T_{j i}, \quad I_{i j}=I_{j i}, \quad T_{i i}=F_{i i}=0.5 \\
& \text { and } I_{i i}=0, \quad \text { for any } i, j \in\{1,2, \ldots, n\} .
\end{aligned}
$$

Definition 3.6. Let $\tilde{A}=\left(\tilde{a}_{i j}\right)_{n \times n}$ be an NFPR, where $\tilde{a}_{i j}=\left\langle T_{\tilde{a}_{i j}}\left(x_{i j}\right), I_{\tilde{a}_{i j}}\left(x_{i j}\right), F_{\tilde{a}_{i j}}\left(x_{i j}\right)\right\rangle$ is represented as $\tilde{a}_{i j}=\left\langle T_{i j}, I_{i j}, F_{i j}\right\rangle . \tilde{A}$ is additive consistent if it satisfies the following additive transitivity:

$$
\begin{aligned}
& T_{i j}\left(1-I_{i j}\right)+T_{j k}\left(1-I_{j k}\right)+T_{k i}\left(1-I_{k i}\right) \\
& \quad=T_{k j}\left(1-I_{k j}\right)+T_{j i}\left(1-I_{j i}\right)+T_{i k}\left(1-I_{i k}\right),
\end{aligned}
$$

for any $i, j, k \in\{1,2, \ldots, n\}$.

As $T_{i j}=F_{j i}, F_{i j}=T_{j i}, I_{i j}=I_{j i}$ for any $i, j \in$ $\{1,2, \ldots, n\}$, it follows from Eq. (6) that:

$$
\begin{aligned}
& F_{i j}\left(1-I_{i j}\right)+F_{j k}\left(1-I_{j k}\right)+F_{k i}\left(1-I_{k i}\right) \\
& \quad=F_{k j}\left(1-I_{k j}\right)+F_{j i}\left(1-I_{j i}\right)+F_{i k}\left(1-I_{i k}\right),
\end{aligned}
$$

for any $i, j, k \in\{1,2, \ldots, n\}$.

In addition, if there exists $I_{i j}=0$ in all SVNESs, $\tilde{a}_{i j}=\left\langle T_{i j}, I_{i j}, F_{i j}\right\rangle$ for any $i, j, k \in\{1,2, \ldots, n\}$, then the NFPR $\tilde{A}=\left(\tilde{a}_{i j}\right)_{n \times n}$ is equivalent to the IPR, and Eqs. (6) and (7) reduce to Eqs. (3) and (4), respectively. According to Definition 3.4, we can get the following property:

Theorem 3.2. An NFPR $\tilde{A}=\left(\tilde{a}_{i j}\right)_{n \times n}$ with $\tilde{a}_{i j}=$ $\left\langle T_{i j}, I_{i j}, F_{i j}\right\rangle$ is additive consistent if and only if $S\left(\tilde{a}_{i j}\right)=S\left(\tilde{a}_{i k}\right)-S\left(\tilde{a}_{j k}\right)$ for any $i, j, k \in\{1,2, \ldots, n\}$.

Theorem 3.2 provides an easy method to tell whether an NFPR satisfies the additive consistency or not.

According to Theorem 3.2, it is obvious that the additive consistency for an NFPR is too strict to be satisfied in the realistic world. Thus, we give another definition of consistency for NFPR, namely, acceptable consistency, which can be easily accepted.

Definition 3.7. Let $\tilde{A}=\left(\tilde{a}_{i j}\right)_{n \times n}$ be an NFPR, $S\left(\tilde{a}_{i j}\right)$ the score function of $\tilde{a}_{i j}$ obtained by directly comparing alternatives $x_{i}$ and $x_{j}$, and $S\left(\tilde{a}_{i k}\right)-S\left(\tilde{a}_{j k}\right)$ the difference between the scores of neutrosophic fuzzy preference values derived by comparing them with an intermediate alternative, $x_{k}$, where $k \neq i, j$. Then, the Absolute Mean Deviation (AMD) $\varepsilon_{i j}$ is defined as: 


$$
\varepsilon_{i j}=\frac{1}{n-2} \sum_{k=1, k \neq i, j}^{n}\left|S\left(\tilde{a}_{i j}\right)-S\left(\tilde{a}_{i k}\right)+S\left(\tilde{a}_{j k}\right)\right| .
$$

According to Definition 3.4 and Eq. (5):

$$
S\left(\tilde{a}_{i k}\right)=\left(T_{i k}-F_{i k}\right)\left(1-I_{i k}\right),
$$

and:

$$
S\left(\tilde{a}_{i k}\right)=\left(F_{k i}-T_{k i}\right)\left(1-I_{k i}\right)=-S\left(\tilde{a}_{k i}\right) .
$$

Thus, Eq. (8) is equal to Eq. (9) in the following:

$$
\varepsilon_{i j}=\frac{1}{n-2} \sum_{k=1, k \neq i, j}^{n}\left|S\left(\tilde{a}_{i j}\right)+S\left(\tilde{a}_{j k}\right)+S\left(\tilde{a}_{k i}\right)\right| .
$$

Definition 3.8. Let $\tilde{A}=\left(\tilde{a}_{i j}\right)_{n \times n}$ be an NFPR, then $C I(\tilde{A})$ is the consistency index for $\tilde{A}$ as follows:

$$
\begin{aligned}
C I & (\tilde{A})=1-\frac{1}{3 n(n-1)(n-2)} \\
& \sum_{i=1}^{n} \sum_{j=1, j \neq i}^{n} \sum_{k=1, k \neq i, j}^{n}\left|S\left(\tilde{r}_{i j}\right)+S\left(\tilde{r}_{j k}\right)+S\left(\tilde{r}_{k i}\right)\right| .
\end{aligned}
$$

For convenience, we let:

$$
\dot{S}(\tilde{A})=\sum_{i=1}^{n} \sum_{j=1, j \neq i}^{n} \sum_{k=1, k \neq i, j}^{n}\left|S\left(\tilde{r}_{i j}\right)+S\left(\tilde{r}_{j k}\right)+S\left(\tilde{r}_{k i}\right)\right|,
$$

then Eq. (10) can be written as:

$$
C I(\tilde{A})=1-\frac{1}{3 n(n-1)(n-2)} \dot{S}(\tilde{A}) .
$$

It is clear that $0 \leq C I(\tilde{A}) \leq 1$ and the greater the $C I(\tilde{A})$, the more consistent the $\tilde{A}$. In particular, if $C I(\tilde{A})=1$, which is equal to $\varepsilon_{i j}=0$ for any $i, j \in\{1,2, \ldots, n\}$, the NFPR $\tilde{A}$ is additive consistent; otherwise, $\tilde{A}$ does not satisfy the additive consistency.

From Definition 3.4, we conclude that:

$$
\begin{aligned}
S\left(\tilde{r}_{i j}\right) & +S\left(\tilde{r}_{j k}\right)+S\left(\tilde{r}_{k i}\right)=T_{i j}\left(1-I_{i j}\right) \\
& -F_{i j}\left(1-I_{i j}\right)+T_{j k}\left(1-I_{j k}\right) \\
& -F_{j k}\left(1-I_{j k}\right)+T_{k i}\left(1-I_{k i}\right)-F_{k i}\left(1-I_{k i}\right) .
\end{aligned}
$$

Since $T_{i j}=F_{j i}, F_{i j}=T_{j i}$ in the NFPR $\tilde{A}$ for any $i, j \in\{1,2, \ldots, n\}$, Eq. (10) can be represented as:

$$
C I(\tilde{A})=1-\frac{1}{3 n(n-1)(n-2)} \dot{T}(\tilde{A}),
$$

where:

$$
\begin{aligned}
\dot{T} & (\tilde{A})=\sum_{i=1}^{n} \sum_{j=1, j \neq i}^{n} \sum_{k=1, k \neq i, j}^{n} \mid T_{i j}\left(1-I_{i j}\right) \\
& +T_{j k}\left(1-I_{j k}\right)+T_{k i}\left(1-I_{k i}\right)-T_{j i}\left(1-I_{j i}\right) \\
& -T_{k j}\left(1-I_{k j}\right)-T_{i k}\left(1-I_{i k}\right) \mid .
\end{aligned}
$$

and as $C I(\tilde{A})=1-\frac{1}{3 n(n-1)(n-2)} \dot{F}(\tilde{A})$, where:

$$
\begin{aligned}
& \dot{F}(\tilde{A})=\sum_{i=1}^{n} \sum_{j=1, j \neq i}^{n} \sum_{k=1, k \neq i, j}^{n} \mid F_{j i}\left(1-I_{j i}\right) \\
& \quad+F_{k j}\left(1-I_{k j}\right)+F_{i k}\left(1-I_{i k}\right)-F_{i j}\left(1-I_{i j}\right) \\
& \quad-F_{j k}\left(1-I_{j k}\right)-F_{k i}\left(1-I_{k i}\right) \mid .
\end{aligned}
$$

Since the additive consistency for NFPR is too strict, we introduce the following acceptable consistency in order to check the consistency levels in GDM problem.

Definition 3.9. Let $C I(\tilde{A})$ be the consistency index for an NFPR $\tilde{A}=\left(\tilde{a}_{i j}\right)_{n \times n}$ and $0 \leq \theta \leq 1$ be an acceptable consistency threshold, then NFPR $\tilde{A}$ is acceptably consistent if it satisfies $C I(\tilde{A}) \geq \theta$; otherwise, $\tilde{A}$ is an unacceptably consistent NFPR. In addition, the greater the value of $\theta$, the stricter of the consistency can be for $\tilde{A}$. The acceptable consistency is especially equal to the additive consistency when $\theta=1$. DMs could choose an appropriate value of $\theta$ to construct a reasonably acceptable consistency. In general, it is suggested that DMs should set $\theta \in[0.5,1]$, and the value of $\theta$ should be appropriately reduced with a large value of $n$.

Definition 3.10. Let:

$$
\tilde{A}=\left(\tilde{a}_{i j}\right)_{n \times n}=\left(\left\langle T_{a i j}, I_{a i j}, F_{a i j}\right\rangle\right)_{n \times n},
$$

and:

$$
\tilde{B}=\left(\tilde{b}_{i j}\right)_{n \times n}=\left(\left\langle T_{b i j}, I_{b i j}, F_{b i j}\right\rangle\right)_{n \times n},
$$

be two NFPRs, then the Mean Absolute Deviation (MAD) between $\tilde{A}$ and $\tilde{B}$ can be computed as follows:

$$
\begin{aligned}
& M A D(\tilde{A}, \tilde{B})=\frac{1}{2 n(n-1)} \\
& \quad \sum_{i=1}^{n} \sum_{j=1, j \neq i}^{n}\left(\left|T_{a i j}\left(1-I_{a i j}\right)-T_{b i j}\left(1-I_{b i j}\right)\right|\right. \\
& \left.\quad+\left|F_{a i j}\left(1-I_{a i j}\right)-F_{b i j}\left(1-I_{b i j}\right)\right|\right) .
\end{aligned}
$$

It is obvious that the smaller is the $M A D(\tilde{A}, \tilde{B})$, 
the closer $\tilde{A}$ is to $\tilde{B}$. In particular, $\tilde{A}=\tilde{B}$ when $M A D(\tilde{A}, \tilde{B})=0$.

Theorem 3.3. If $M A D(\tilde{A}, \tilde{B})$ is the MAD between two NFPRs $\tilde{A}=\left(\tilde{a}_{i j}\right)_{n \times n}=\left(\left\langle T_{a i j}, I_{a i j}, F_{a i j}\right\rangle\right)_{n \times n}$ and $\tilde{B}=\left(\tilde{b}_{i j}\right)_{n \times n}=\left(\left\langle T_{b i j}, I_{b i j}, F_{b i j}\right\rangle\right)_{n \times n}$, it should satisfy the following properties:

(a) $0 \leq \operatorname{MAD}(\tilde{A}, \tilde{B}) \leq 1$;

(b) $M A D(\tilde{A}, \tilde{B})=\operatorname{MAD}(\tilde{B}, \tilde{A})$;

(c) The MAD between $\tilde{A}$ and $\tilde{B}$ can be written as:

$$
\begin{array}{r}
M A D(\tilde{A}, \tilde{B})=\frac{1}{n(n-1)} \sum_{i=1}^{n} \sum_{j=1, j \neq i}^{n} \\
\left|T_{a i j}\left(1-I_{a i j}\right)-T_{b i j}\left(1-I_{b i j}\right)\right| .
\end{array}
$$

\section{An approach for improving consistency of NFPRs}

MCGDM problems with vague information are widespread in the real world and are therefore the foci of many scholars [56-60]. Different extensions of PRs have been developed because of their effectiveness in expressing the DM's preferences $[32,48,61]$. Although in the process of MCGDM, the most common method to improve an NFPR with unacceptable consistency is to let DMs update the original information, it is hard for the DMs to adjust their original judgments to real life. To solve this conundrum, we have devised a method to improve the consistency of NFPRs, which retains the original information as much as possible. The innovations of this paper include: (i) NFPRs to help DMs express their preferences more accurately, (ii) a method to improve consistency of NFPRs, and (iii) a novel method to obtain the weights of criteria based on the preference values of DMs in MCGDM problems.

Definition 4.1. Let $\tilde{A}=\left(\tilde{a}_{i j}\right)_{n \times n}$ be an NFPR with $\tilde{a}_{i j}=\left\langle T_{i j}, I_{i j}, F_{i j}\right\rangle$, then NFPR $\hat{A}=\left(\hat{a}_{i j}\right)_{n \times n}$ is an additive consistent NFPR if $\hat{a}_{i j}=\left\langle\hat{T}_{i j}, \hat{I}_{i j}, \hat{F}_{i j}\right\rangle$ satisfies $\hat{T}_{i j} \geq 0, \hat{I}_{i j} \geq 0$ and:

$$
\begin{aligned}
& \hat{T}_{i j}\left(1-\hat{I}_{i j}\right)=\frac{1}{2 n}\left(\sum_{l=1}^{n}\left(T_{i l}\left(1-I_{i l}\right)-T_{j l}\left(1-I_{j l}\right)\right)\right. \\
& \left.\quad-\sum_{l=1}^{n}\left(T_{l i}\left(1-I_{l i}\right)-T_{l j}\left(1-I_{l j}\right)\right)\right) \\
& \quad+0.5\left(T_{i j}\left(1-I_{i j}\right)+T_{j i}\left(1-I_{j i}\right)\right),
\end{aligned}
$$

$$
\begin{aligned}
\hat{F}_{i j}\left(1-\hat{I}_{i j}\right)=\frac{1}{2 n}\left(\sum_{l=1}^{n}\left(F_{i l}\left(1-I_{i l}\right)-F_{j l}\left(1-I_{j l}\right)\right)\right. \\
\left.-\sum_{l=1}^{n}\left(F_{l i}\left(1-I_{l i}\right)-F_{l j}\left(1-I_{l j}\right)\right)\right) \\
+0.5\left(F_{i j}\left(1-I_{i j}\right)+F_{j i}\left(1-I_{j i}\right)\right), \\
\hat{I}_{i j}= \begin{cases}1-\hat{T}_{i j}\left(1-\hat{I}_{i j}\right) / T_{i j}, & \hat{T}_{i j}\left(1-\hat{I}_{i j}\right) / T_{i j} \leq 1 \\
0, & \text { otherwise }\end{cases}
\end{aligned}
$$

for any $i \geq j \in\{1,2, \ldots, n\}$,

$\hat{T}_{i j}= \begin{cases}T_{i j}, & \hat{T}_{i j}\left(1-\hat{I}_{i j}\right) / T_{i j} \leq 1 \\ \hat{T}_{i j}\left(1-\hat{I}_{i j}\right), & \text { otherwise }\end{cases}$

for any $i \geq j \in\{1,2, \ldots, n\}$,

$$
\hat{F}_{i j}=\frac{\hat{F}_{i j}\left(1-\hat{I}_{i j}\right)}{1-\hat{I}_{i j}},
$$

for any $i \geq j \in\{1,2, \ldots, n\}$,

$$
\hat{I}_{i j}=\hat{I}_{j i}, \quad \hat{T}_{i j}=\hat{F}_{j i}, \quad \hat{F}_{i j}=\hat{T}_{j i}
$$

for any $i<j \in\{1,2, \ldots, n\}$.

In addition, if there exits $\hat{T}_{i j}<0$ for some $i, j \in$ $\{1,2, \ldots, n\}$ in $\hat{A}=\left(\hat{a}_{i j}\right)_{n \times n}$, it is not an NFPR. In this case, all of the values of $\hat{T}_{i j}$ should be converted to an interval $[0,1]$. Therefore, we give the following definition of the converted values according to the reference [29].

Definition 4.2. Let $\hat{A}=\left(\hat{a}_{i j}\right)_{n \times n}$ with $\hat{a}_{i j}=$ $\left\langle\hat{T}_{i j}, \hat{I}_{i j}, \hat{F}_{i j}\right\rangle$ be the matrix obtained from the NFPR $\tilde{A}=\left(\tilde{a}_{i j}\right)_{n \times n}$ based on Definition 4.1. Then, matrix $\tilde{A}^{\prime}=\left(\tilde{a}_{i j}^{\prime}\right)_{n \times n}$ is called a rectified matrix with $\tilde{a}_{i j}^{\prime}=$ $\left\langle\tilde{T}_{i j}^{\prime}, \tilde{I}_{i j}^{\prime}, \tilde{F}_{i j}^{\prime}\right\rangle$ if the converted values are as follows:

$$
\begin{gathered}
\tilde{T}_{i j}^{\prime}\left(1-\tilde{I}_{i j}^{\prime}\right)=\frac{\hat{T}_{i j}\left(1-\hat{I}_{i j}\right)+t}{1+2 t}, \\
\tilde{F}_{i j}^{\prime}\left(1-\tilde{I}_{i j}^{\prime}\right)=\frac{\hat{F}_{i j}\left(1-\hat{I}_{i j}\right)+t}{1+2 t},
\end{gathered}
$$

for any $i, j \in\{1,2, \ldots, n\}$,

where:

$$
t= \begin{cases}0, & \hat{T}_{i j}\left(1-\hat{I}_{i j}\right) \geq 0 \\ \max \left\{\left|\hat{T}_{i j}\left(1-\hat{I}_{i j}\right)\right|\right\}, & \hat{T}_{i j}\left(1-\hat{I}_{i j}\right)<0\end{cases}
$$

for any $i, j \in\{1,2, \ldots, n\}$, 


$$
\tilde{I}_{i j}^{\prime}= \begin{cases}1-\tilde{T}^{\prime}{ }_{i j}\left(1-\tilde{I}_{i j}^{\prime}\right) / \hat{T}_{i j}, & \tilde{T}_{i j}^{\prime}\left(1-\tilde{I}_{i j}^{\prime}\right) / \hat{T}_{i j} \leq 1 \\ 0, & \text { otherwise }\end{cases}
$$

for any $i \geq j \in\{1,2, \ldots, n\}$,

$$
\tilde{T}_{i j}^{\prime}= \begin{cases}\hat{T}_{i j}, & \tilde{T}^{\prime}{ }_{i j}\left(1-\tilde{I}^{\prime}{ }_{i j}\right) / \hat{T}_{i j} \leq 1 \\ \tilde{T}^{\prime}{ }_{i j}\left(1-\tilde{I}^{\prime}{ }_{i j}\right), & \text { otherwise }\end{cases}
$$

for any $i \geq j \in\{1,2, \ldots, n\}$,

$$
\begin{aligned}
& \tilde{F}_{i j}^{\prime}=\frac{\tilde{F}_{i j}^{\prime}\left(1-\tilde{I}_{j i}^{\prime}\right)}{1-\tilde{I}_{j i}^{\prime}}, \quad \text { for any } i \geq j \in\{1,2, \ldots, n\} \\
& \tilde{T}^{\prime}{ }_{i j}=\tilde{F}_{j i}^{\prime}, \quad \tilde{F}_{i j}^{\prime}=\tilde{T}_{j i}^{\prime}, \quad \tilde{I}_{i j}^{\prime}=\tilde{I}^{\prime}{ }_{j i} \\
& \text { for any } i<j \in\{1,2, \ldots, n\} .
\end{aligned}
$$

Theorem 4.1. Let $\tilde{A}=\left(\tilde{a}_{i j}\right)_{n \times n}$ be an NFPR and the derived matrix $\tilde{A}^{\prime}=\left(\tilde{a}_{i j}^{\prime}\right)_{n \times n}$ be converted based on Eqs. (15) and (16), then $\tilde{A}^{\prime}=\left(\tilde{a}_{i j}^{\prime}\right)_{n \times n}$ is an additive consistent NFPR.

Theorem 4.2. Let $\tilde{A}=\left(\tilde{a}_{i j}\right)_{n \times n}$ be an additive consistent NFPR and $\tilde{A}^{\prime}=\left(\tilde{a}_{i j}^{\prime}\right)_{n \times n}$ the derived matrix obtained from $\tilde{A}$ based on Definition 4.2, then $\tilde{A}=\tilde{A}^{\prime}$.

Definition 4.3. Let $\tilde{A}=\left(\tilde{a}_{i j}\right)_{n \times n}$ be an NFPR with $\tilde{a}_{i j}=\left\langle\tilde{T}_{i j}, \tilde{I}_{i j}, \tilde{F}_{i j}\right\rangle$ and $\tilde{A}^{\prime}=\left(\tilde{a}_{i j}^{\prime}\right)_{n \times n}$ be the derived additive consistent matrix where $\tilde{a}_{i j}^{\prime}=\left\langle\tilde{T}_{i j}^{\prime}, \tilde{I}_{i j}^{\prime}, \tilde{F}_{i j}^{\prime}\right\rangle$, then $\tilde{A}(\lambda)=\left(\tilde{a}_{i j}(\lambda)\right)$ is a weighted averaging matrix if $\tilde{a}_{i j}(\lambda)=\left\langle\tilde{T}_{i j}(\lambda), \tilde{I}_{i j}(\lambda), \tilde{F}_{i j}(\lambda)\right\rangle$ satisfies:

$$
\begin{aligned}
& \tilde{T}_{i j}(\lambda)\left(1-\tilde{I}_{i j}(\lambda)\right)=(1-\lambda) \tilde{T}_{i j}\left(1-\tilde{I}_{i j}\right) \\
& +\lambda \tilde{T}_{i j}^{\prime}\left(1-\tilde{I}_{i j}^{\prime}\right), \\
& \tilde{I}_{i j}(\lambda)=\left\{\begin{aligned}
& 1-\tilde{T}_{i j}(\lambda)\left(1-\tilde{I}_{i j}(\lambda)\right) / \tilde{T}_{i j}, \\
& \tilde{T}_{i j}(\lambda)\left(1-\tilde{I}_{i j}(\lambda)\right) / \tilde{T}_{i j} \leq 1 \\
& 0, \text { otherwise }
\end{aligned}\right.
\end{aligned}
$$

for any $i \geq j \in\{1,2, \ldots, n\}$,

$$
\tilde{T}_{i j}(\lambda)=\left\{\begin{array}{l}
\tilde{T}_{i j}, \quad \tilde{T}_{i j}(\lambda)\left(1-\tilde{I}_{i j}(\lambda)\right) / \tilde{T}_{i j} \leq 1 \\
\tilde{T}_{i j}(\lambda)\left(1-\tilde{I}_{i j}(\lambda)\right), \quad \text { otherwise }
\end{array}\right.
$$

for any $i \geq j \in\{1,2, \ldots, n\}$,

$$
\begin{aligned}
& \tilde{F}_{i j}(\lambda)=\frac{\tilde{F}_{i j}(\lambda)\left(1-\tilde{I}_{j i}(\lambda)\right)}{1-\tilde{I}_{j i}(\lambda)}, \\
& \text { for any } i \geq j \in\{1,2, \ldots, n\}, \\
& \tilde{T}_{i j}(\lambda)=\tilde{F}_{j i}(\lambda), \quad \tilde{F}_{i j}(\lambda)=\tilde{T}_{j i}(\lambda), \\
& \tilde{I}_{i j}(\lambda)=\tilde{I}_{j i}(\lambda), \\
& \text { for any } i<j \in\{1,2, \ldots, n\} .
\end{aligned}
$$

Theorem 4.3. If $\tilde{A}(\lambda)=\left(\tilde{a}_{i j}(\lambda)\right)$ is a weighted averaging matrix obtained from an original NFPR $\tilde{A}=\left(\tilde{a}_{i j}\right)_{n \times n}$ and its derived additive consistent NFPR $\tilde{A}^{\prime}=\left(\tilde{a}_{i j}^{\prime}\right)_{n \times n}$ based on Definition 4.3, then $\tilde{A}(\lambda)$ is still an NFPR.

Theorem 4.4. Let $0 \leq \theta \leq 1$ be an acceptable consistency threshold, and $\tilde{A}=\left(\tilde{a}_{i j}\right)_{n \times n}$ and $\tilde{A}^{\prime}=$ $\left(\tilde{a}_{i j}^{\prime}\right)_{n \times n}$ be the original NFPR and the derived consistent NFPR, respectively, then matrix $\tilde{A}(\lambda)=\left(\tilde{a}_{i j}(\lambda)\right)$ is an acceptably consistent NFPR if $\frac{\theta-C I(\tilde{A})}{1-C I(\tilde{A})} \leq \lambda \leq 1$.

Based on the above definitions, the process to construct an acceptably consistent NFPR is summarized as follows:

Step 1. Construct the additive consistent NFPR $\hat{A}=\left(\hat{a}_{i j}\right)_{n \times n}$ based on an NFPR $\tilde{A}=\left(\tilde{a}_{i j}\right)_{n \times n}$ according to Eq. (15);

Step 2. Convert $\hat{A}=\left(\hat{a}_{i j}\right)_{n \times n}$ into a rectified matrix $\tilde{A}^{\prime}=\left(\tilde{a}_{i j}^{\prime}\right)_{n \times n}$ as per Eq. (16);

Step 3. Calculate the weight $\lambda_{0}=\frac{\theta-C I(\tilde{A})}{1-C I(\tilde{A})}$, where $0 \leq \theta \leq 1$ is the acceptable consistency threshold decided by the DM;

Step 4. As per Definition 4.3, build the improved acceptably consistent NFPR $\tilde{A}(\lambda)=\left(\tilde{a}_{i j}(\lambda)\right)$.

In Definitions 3.10 and 4.3 , we can clearly see that the MAD value reflects similarity between two NFPRs and it is appropriate for several individual NFPRs under one criterion. For several NFPRs under different criteria, the bigger MAD value between one NFPR and its acceptably consistent NFPR implies the higher consistency of this NFPR, which implies that DMs are more consistent on their preferences for this criterion. Since the criterion that has less disagreement deserves higher weight, it is reasonable to apply MAD value for solving MCDM problems. Therefore, we apply the MAD value to obtain the weights of criteria in MCGDM problem. 


\section{An MCGDM method based on the consistency of NFPRs}

In this section, we propose a systematic MCGDM method under the environment of NFPRs, including the measuring consistency for the original preference matrices; thus, we improve the consistency of the NFPRs with unacceptable consistency, integrate them, and rank the alternatives.

\subsection{Aggregation operators for NFPRs}

The aim of this section is to develop some aggregation operators which are essential for aggregating the NFPRs in the MCGDM problems.

Definition 5.1. Let:

$$
\tilde{A}_{z}=\left(\tilde{a}_{z i j},\right)_{n \times n}
$$

be an NFPR, where

$$
\tilde{a}_{z i j}=\left\langle T_{\tilde{a}_{z i j}}, I_{\tilde{a}_{z i j}}, F_{\tilde{a}_{i j}}\right\rangle,
$$

and:

$$
W=\left(w_{1}, w_{2}, \ldots, w_{k}\right)^{T},
$$

be the weight vector with $w_{z} \in[0,1]$ and $\sum_{z=1}^{k} w_{z}=$ 1, then the Neutrosophic Fuzzy Preference Relation Weighted Averaging (NFPRWA) operator is as follows:

$$
\begin{gathered}
N F P R W A\left(\tilde{A}_{1}, \tilde{A}_{2}, \ldots, \tilde{A}_{k}\right)=\left(\bigoplus_{z=1}^{k}\left(w_{z} \tilde{a}_{z i j}\right)\right)_{n \times n} \\
=\left(\left\langle\sum_{z=1}^{k} w_{z} T_{\tilde{a} z i j}, \sum_{z=1}^{k} w_{z} I_{\tilde{a} z i j}, \sum_{z=1}^{k} w_{z} F_{\tilde{a} z i j}\right\rangle\right)_{n \times n}
\end{gathered}
$$

Definition 5.2 Let $\tilde{A}_{z}=\left(\tilde{a}_{z i j}\right)_{n \times n}$ be an NFPR with $\tilde{a}_{z i j}=\left\langle T_{\tilde{a}_{z i j}}, I_{\tilde{a}_{z i j}}, F_{\tilde{a}_{i j}}\right\rangle$, then the Neutrosophic Fuzzy Preference Relation Induced Ordered Weighted Averaging (NFPRIOWA) operator is defined as:

$$
\begin{gathered}
\text { NPRIOWA }\left(\left\langle H_{1}, \tilde{A}_{\sigma(1)}\right\rangle,\left\langle H_{2}, \tilde{A}_{\sigma(2)}\right\rangle, \ldots,\right. \\
\left.\left\langle H_{k}, \tilde{A}_{\sigma(k)}\right\rangle\right)=\left(\bigoplus_{z=1}^{k}\left(w_{z} \tilde{a}_{\sigma(z) i j}\right)\right)_{n \times n} \\
=\left(\left\langle\sum_{z=1}^{k} w_{z} T_{\tilde{a} \sigma(z) i j}, \sum_{z=1}^{k} w_{z} I_{\tilde{a} \sigma(z) i j},\right.\right. \\
\left.\left.\sum_{z=1}^{k} w_{z} F_{\tilde{a} \sigma(z) i j}\right\rangle\right)_{n \times n},
\end{gathered}
$$

where $\left\langle H_{1}, \tilde{A}_{\sigma(1)}\right\rangle,\left\langle H_{2}, \tilde{A}_{\sigma(2)}\right\rangle, \ldots,\left\langle H_{k}, \tilde{A}_{\sigma(k)}\right\rangle$ is a set of OWA pairs, $W=\left(w_{1}, w_{2}, \ldots, w_{k}\right)^{T}$ is the asso- ciated weight vector with $w_{z} \in[0,1]$ and $\sum_{z=1}^{k} w_{z}=1, \sigma$ is the permutation of $\{1,2, \ldots, k\}$ with $H_{\sigma(z)} \geq H_{\sigma(z+1)}$ for any $z \in\{1,2, \ldots, k\}$, and $\tilde{A}_{\sigma(z)}$ is reordering of $\tilde{A}_{z}$ as per the decreasing order of $\left\{H_{1}, H_{2}, \ldots, H_{k}\right\}$.

In particular, if the order of $\tilde{A}_{z}$ is the same as that of $\tilde{A}_{\sigma(k)}$, the NFPRIOWA operator reduces to the NFPRWA operator and if $W=(1 / k, 1 / k, \ldots, 1 / k)^{T}$, the NFPRWA operator is equal to the neutrosophic fuzzy preference relation averaging (NFPRA) operator as:

$$
\begin{gathered}
N F P R A\left(\tilde{A}_{1}, \tilde{A}_{2}, \ldots, \tilde{A}_{k}\right)=\left(\bigoplus_{z=1}^{k}\left(\frac{1}{k} \tilde{a}_{z i j}\right)\right)_{n \times n} \\
=\left(\left\langle\sum_{z=1}^{k} \frac{1}{k} T_{\tilde{a} z i j}, \sum_{z=1}^{k} \frac{1}{k} I_{\tilde{a} z i j}, \sum_{z=1}^{k} \frac{1}{k} F_{\tilde{a} z i j}\right\rangle\right)_{n \times n} .
\end{gathered}
$$

In addition, we define the ordering inducing value $H_{z}$, which accounts for both the MAD value $M A D\left(\tilde{A}_{z}, \tilde{A}_{z}(\lambda)\right)$ and the consistency index value $C I\left(\tilde{A}_{z}(\lambda)\right)$, as follows:

$$
H_{z}=\frac{1}{2} C I\left(\tilde{A}_{z}(\lambda)\right)+\frac{1}{2}\left(1-M A D\left(\tilde{A}_{z}, \tilde{A}_{z}(\lambda)\right)\right),
$$

where $p=1,2, \ldots, k$.

On the basis of $H_{z}$, the associated weight vector is defined as:

$$
w_{t}=\left\{\begin{array}{c}
\left(H_{\sigma(1)} / \sum_{z=1}^{k} H_{\sigma(1)}\right)^{\beta}, \mathrm{t}=1 \\
\left(\sum_{z=1}^{t} H_{\sigma(1)} / \sum_{z=1}^{k} H_{\sigma(1)}\right)^{\beta} \\
-\left(\sum_{z=1}^{t-1} H_{\sigma(1)} / \sum_{z=1}^{k} H_{\sigma(1)}\right)^{\beta}, \\
\mathrm{t}=2,3, \ldots, k
\end{array}\right.
$$

where $0 \leq \beta \leq 1$ is a parameter controlling the value of $H_{1}$; if $\beta=1$, Eq. (19) is based on the normalized induced value aggregation method for the NFPRs, and if $\beta=0, \mathrm{Eq}$. (19) is based on the maximum inducing aggregation method for the NFPRs. It is obvious that if $H_{\sigma(1)} \geq H_{\sigma(2)} \geq \ldots \geq H_{\sigma(k)}$, then $w_{\sigma(1)} \geq w_{\sigma(2)} \geq$ $\ldots \geq w_{\sigma(k)}$, so the higher the value $H_{z}$, the greater the weight $w_{z}$ is.

\subsection{An MCGDM method with NFPRs}

Based on the above definitions, we have detailed the steps of an MCGDM method with the NFPRs as shown below. The scenario is that $t$ experts are asked to give their own preference values over $n$ alternatives under $k$ criteria.

Step 1. Compute the consistency level $C I\left(\tilde{A}_{z_{p}}\right)$ 
for NFPRs $\tilde{A}_{z_{p}}=\left(\tilde{a}_{z_{p} i j}\right)_{n \times n}$, where $\tilde{a}_{z_{p} i j}=$ $\left\langle T_{\tilde{a}_{z_{p} i j}}, I_{\tilde{a}_{z_{p} i j}}, F_{\tilde{a} p_{i j}}\right\rangle$ and $\tilde{A}_{z_{p}}$ is the preference value matrix provided by the pth DM under the zth attribute for alternatives based on Eqs. (10)-(12), in which $i, j=1,2, \ldots, n, p=1,2, \ldots, t$, and $z=$ $1,2, \ldots, k$;

Step 2. For every $\tilde{A}_{z_{p}}$, if $C I\left(\tilde{A}_{z_{p}}\right) \geq \theta$, let $\tilde{A}_{z_{p}}(\lambda)=\tilde{A}_{z_{p}}$; otherwise, return the original matrix $\tilde{A}_{z_{p}}$ to the DMs and go back to Step 1. If it is not feasible for the DMs to update $\tilde{A}_{z_{p}}$, then calculate the weighted averaging matrix $\tilde{A}_{z_{p}}(\lambda)=\left(\tilde{a}_{z_{p} i j}(\lambda)\right)$ as per Eq. (17);

Step 3. Aggregate NFPRs $\tilde{A}_{z_{p}}(\lambda)$ for any $z \in$ $\{1,2, \ldots, k\}$ into $k$ NFPRs $\tilde{A}_{z}=\left(\tilde{a}_{z i j}\right)_{n \times n}$ using the NFPRWA operator with given weights of $t$ experts, as per Eq. (18);

Step 4. Calculate the MAD value $\operatorname{MAD}\left(\tilde{A}_{z}\right.$, $\tilde{A}_{z}(\lambda)$, where $z=1,2, \ldots, k$ according to Eqs. (13) and (14);

Step 5. Compute the ordering inducing value $H_{z}$ by combining both the $C I\left(\tilde{A}_{z}(\lambda)\right)$ and $M A D$ $\left(\tilde{A}_{z}, \tilde{A}_{z}(\lambda)\right)$, based on Eq. (21);

Step 6. Get the value of associated weights $W=$ $\left(w_{1}, w_{2}, \ldots, w_{k}\right)^{T}$ according to Eq. (22);

- Step 7. Aggregate all the NFPRs $\tilde{A}_{z}(\lambda)$ for any $z \in\{1,2, \ldots, k\}$ into a group NFPR $\tilde{A}=\left(\tilde{a}_{i j}\right)_{n \times n}$ using the NFPRIOWA operator as per Eq. (19);

Step 8. Calculate the score function of $\tilde{a}_{i j}$ for any $i, j \in\{1,2, \ldots, n\}$, denoted by $S\left(\tilde{a}_{i j}\right)$, as per Definition 3.4, and get $S\left(A_{i}\right)=\sum_{j=1}^{n} S\left(\tilde{a}_{i j}\right)$ to rank the alternatives. If there exists $S\left(A_{i}\right)=S\left(\tilde{a}_{j}\right)$, where $i \neq j \in\{1,2, \ldots, n\}$, rank the alternatives by computing the sub-score function and sub-accuracy function in the same way.

\subsection{The model of MCGDM method with NFPRs}

In this section, we describe the model of the proposed MCGDM method with NFPRs as depicted in Figure 1.

\section{MCGDM-NFPRs model for medical tourism destination selection}

In this section, we verify flexibility and practicability of our proposed MCGDM-NFPRs model by using Cases 1 and 2, from two different aspects. The backgrounds and data of two cases are described in Section 6.1, followed by Section 6.2 and Section 6.3, which show the computational process and results for two cases, respectively. The results are then discussed in Section 6.4.

\subsection{Background}

The proposed method is conducted to evaluate some medical tourism destinations and select the most appropriate one. The data sources, data processing, and objectives of two cases are different and described as follows:

- Data sources:

- For Case 1, the primary and secondary data were collected from patients, policy makers, doctors, and tours and hospitality managers during the period 2014-2015, including primary data and secondary data. Roy et al. [14] collected the data in order to select the most suitable medical tourism destinations in India. Six experts were invited to give their linguistic decisions for nine cities in India under seven criteria;

- For Case 2 the data were adapted from Wang et al. [29] and the original data were in the form of intuitionistic fuzzy preference values. Four experts were asked to give their own preferences for four alternatives, independently.

- Data processing:

- The original data [14] in Case 1 were in the form of linguistic decision values for each alternative, which were different from the preference values in our method. Therefore, we transformed the experts based decision matrix to 42 neutrosophic preference matrices $\tilde{A}_{z_{p}}=\left(\tilde{a}_{z_{p}} i j\right)_{n \times n}$, where $z=$ $1,2, \ldots, 7, p=1,2, \ldots, 6$, and $i, j=1,2, \ldots, 9$;

- In Case 2, the original data [29] were composed by four intuitionistic preference matrices, representing the independent assessments of the four experts. By comparing every pair among the four alternatives, we adapted them to neutrosophic preference matrices $\tilde{A}_{z}=\left(\tilde{a}_{z i j}\right)_{n \times n}$, where $z=$ $1,2, \ldots, 4$ and $i, j=1,2, \ldots, 4$.

- Objectives:

- To verify the validity and stability of the proposed method in the evaluation and selection of medical tourism destinations, especially the usefulness of MAD value in MCGDM problems, by Case 1 ;

- To demonstrate the advantage of examining and constructing the logical consistency for patienttravelers, and the necessity of using NFPRs, by Case 2;

- To discover the future research directions, according to the results of comparison with other algorithms in the two cases.

\subsection{Illustration of the proposed method (Case 1)}

The data resource has been introduced in Section 6.1. Seven maximizing criteria $\left\{C_{1}, C_{2}, C_{3}, C_{4}, C_{5}, C_{6}, C_{7}\right\}$ 


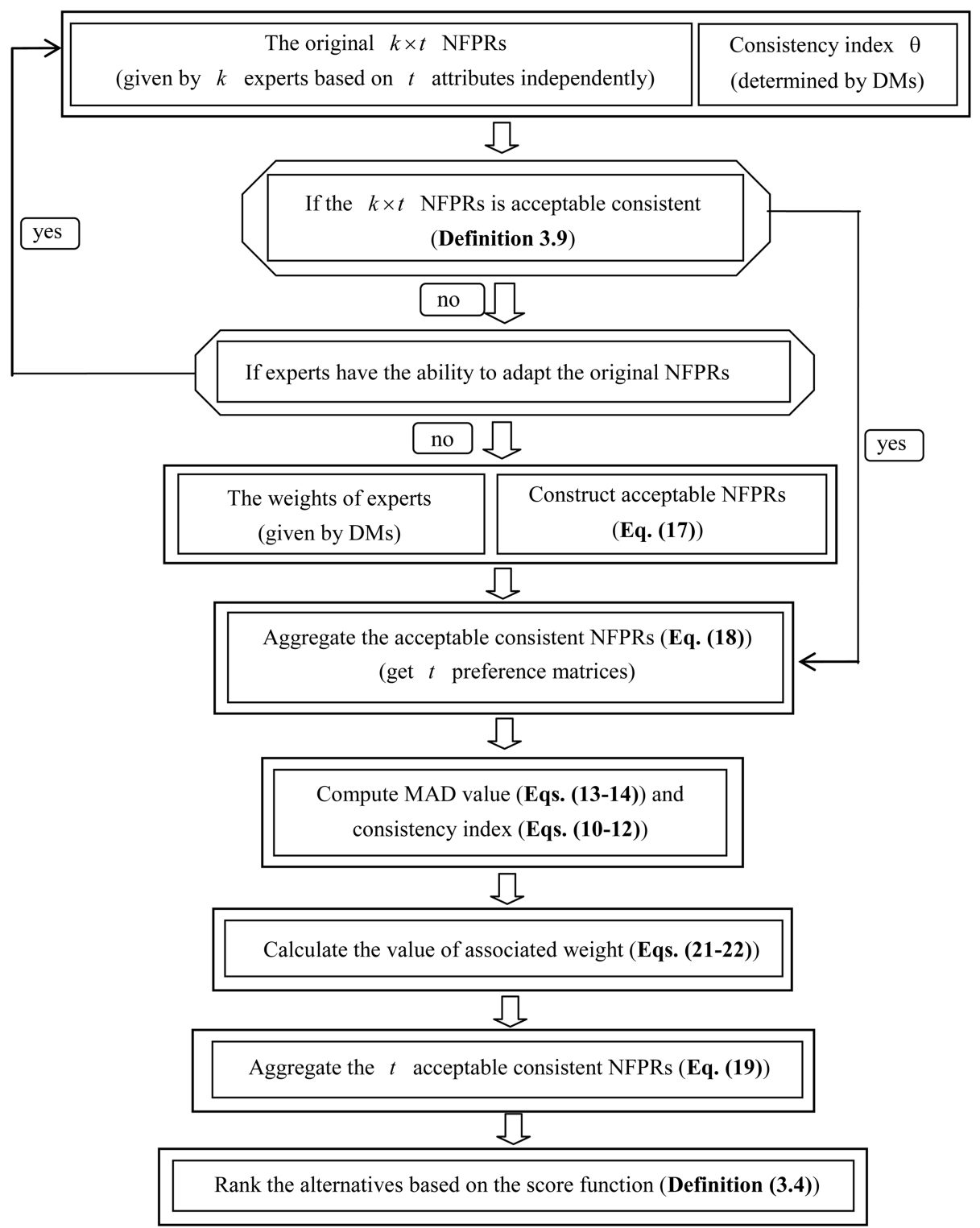

Figure 1. The MCGDM-NFPRs model.

were chosen and divided into three groups: (1) Strengthening of the infrastructure, wherein $C_{1}$ is quality of infrastructure of the medical establishments, $C_{2}$ represents traffic convenience and population statistics, and $C_{3}$ is the information infrastructure and circulation channels; (2) Strengthening of the services for medical tourism in which $C_{4}$ is the supply of skilled technological workers and $C_{5}$ is the quality of medical operators and consultancy centers; and (3) Planning and developing policies for medical tourism, where $C_{6}$ and $C_{7}$ represent progress plan, and corresponding laws and policies, respectively. In consideration of the above criteria, six experts were invited to give their linguistic decisions for nine cities in India including Bangalore, Chennai, Delhi, Hyderabad, Jaipur, Kolkata, Mumbai, Pune, and Kochi.

As described in Section 6.1, the values of experts based decision matrix were expressed by a 9-point scale system, in which 1 stood for "very low", 3 for "low", 5 for "moderate", and 7 and 9 for "high" and "very high," respectively; the values of $2,4,6,8$ were intermediate values. The previous methods required the experts to score every alternative under different attributes using the 9-point system; however, it was quite difficult for a patient-tourist to determine accurate value and since the values were frequently inconsistent, a realistic evaluation of the medical tourism destinations was very difficult.

In order to overcome the above shortcomings, we asked the patient-travelers to give their preference values about every medical tourism destination in the form of a neutrosophic fuzzy number. This neutrosophic preference relation could express thoughts more conveniently and precisely. The original matrices were 


$\tilde{A}_{1}=\left[\begin{array}{ccccc}\langle 0.50,0,0.50\rangle & \langle 0.53,0.13,0.60\rangle & \langle 0.63,0.13,0.53\rangle & \langle 0.63,0.13,0.53\rangle & \\ \langle 0.60,0.13,0.53\rangle & \langle 0.50,0,0.50\rangle & \langle 0.80,0.27,0.57\rangle & \langle 0.80,0.27,0.57\rangle \\ \langle 0.53,0.13,0.63\rangle & \langle 0.57,0.27,0.80\rangle & \langle 0.50,0,0.50\rangle & \langle 0.50,0,0.50\rangle & \\ \langle 0.53,0.13,0.63\rangle & \langle 0.57,0.27,0.80\rangle & \langle 0.50,0,0.50\rangle & \langle 0.50,0,0.50\rangle & \\ \langle 0.59,0.33,0.91\rangle & \langle 0.35,0,0.65\rangle & \langle 0.70,0.55,0.70\rangle & \langle 0.55,0.20,0.70\rangle & \\ \langle 0.59,0.33,0.91\rangle & \langle 0.35,0,0.65\rangle & \langle 0.55,0.20,0.70\rangle & \langle 0.55,0.20,0.70\rangle & \\ \langle 0.35,0,0.65\rangle & \langle 0.31,0,0.69\rangle & \langle 0.59,0.33,0.91\rangle & \langle 0.59,0.33,0.91\rangle & \\ \langle 0.51,0.07,0.56\rangle & \langle 0.55,0.20,0.70\rangle & \langle 0.56,0.07,0.51\rangle & \langle 0.56,0.07,0.51\rangle & \\ \langle 0.35,0,0.65\rangle & \langle 0.31,0,0.69\rangle & \langle 0.59,0.33,0.91\rangle & \langle 0.59,0.33,0.91\rangle & \\ \langle 0.91,0.33,0.59\rangle & \langle 0.91,0.33,0.59\rangle & \langle 0.65,0,0.35\rangle & \langle 0.56,0.07,0.51\rangle & \langle 0.65,0,0.35\rangle \\ \langle 0.65,0,0.35\rangle & \langle 0.65,0,0.35\rangle & \langle 0.69,0,0.31\rangle & \langle 0.70,0.20,0.55\rangle & \langle 0.69,0,0.31\rangle \\ \langle 0.70,0.55,0.70\rangle & \langle 0.70,0.20,0.55\rangle & \langle 0.91,0.33,0.59\rangle & \langle 0.51,0.07,0.56\rangle & \langle 0.91,0.33,0.59\rangle \\ \langle 0.70,0.20,0.55\rangle & \langle 0.70,0.20,0.55\rangle & \langle 0.91,0.33,0.59\rangle & \langle 0.51,0.07,0.56\rangle & \langle 0.91,0.33,0.59\rangle \\ \langle 0.50,0,0.50\rangle & \langle 0.50,0,0.50\rangle & \langle 0.63,0.13,0.53\rangle & \langle 0.57,0.27,0.80\rangle & \langle 0.63,0.13,0.53\rangle \\ \langle 0.50,0,0.50\rangle & \langle 0.50,0,0.50\rangle & \langle 0.63,0.13,0.53\rangle & \langle 0.57,0.27,0.80\rangle & \langle 0.63,0.13,0.53\rangle \\ \langle 0.53,0.13,0.63\rangle & \langle 0.53,0.13,0.63\rangle & \langle 0.50,0,0.50\rangle & \langle 0.63,0.4,0.42\rangle & \langle 0.50,0,0.50\rangle \\ \langle 0.80,0.27,0.57\rangle & \langle 0.80,0.27,0.57\rangle & \langle 0.42,0.4,0.63\rangle & \langle 0.50,0,0.50\rangle & \langle 0.63,0,0.38\rangle \\ \langle 0.53,0.13,0.63\rangle & \langle 0.53,0.13,0.63\rangle & \langle 0.50,0,0.50\rangle & \langle 0.38,0,0.63\rangle & \langle 0.50,0,0.50\rangle\end{array}\right]$

Box I

$$
\tilde{A}=\left[\begin{array}{cccc}
\langle 0.50,0,0.50\rangle & \langle 0.53,0.13,0.66\rangle & \langle 0.60,0.19,0.69\rangle & \langle 0.61,0.10,0.50\rangle \\
\langle 0.66,0.13,0.53\rangle & \langle 0.50,0,0.50\rangle & \langle 0.63,0.15,0.56\rangle & \langle 0.88,0.20,0.54\rangle \\
\langle 0.69,0.19,0.60\rangle & \langle 0.56,0.15,0.63\rangle & \langle 0.50,0,0.50\rangle & \langle 0.59,0.03,0.44\rangle \\
\langle 0.50,0.10,0.61\rangle & \langle 0.54,0.20,0.88\rangle & \langle 0.44,0.03,0.59\rangle & \langle 0.50,0,0.50\rangle \\
\langle 0.61,0.31,0.89\rangle & \langle 0.48,0.18,0.84\rangle & \langle 0.54,0.26,0.84\rangle & \langle 0.58,0.22,0.70\rangle \\
\langle 0.53,0.16,0.69\rangle & \langle 0.43,0.08,0.68\rangle & \langle 0.51,0.17,0.72\rangle & \langle 0.60,0.18,0.63\rangle \\
\langle 0.53,0.12,0.55\rangle & \langle 0.43,0.05,0.64\rangle & \langle 0.53,0.16,0.69\rangle & \langle 0.65,0.21,0.65\rangle \\
\langle 0.58,0.13,0.61\rangle & \langle 0.49,0.10,0.65\rangle & \langle 0.53,0.13,0.64\rangle & \langle 0.65,0.13,0.55\rangle \\
\langle 0.53,0.11,0.53\rangle & \langle 0.49,0.13,0.71\rangle & \langle 0.57,0.22,0.73\rangle & \langle 0.62,0.16,0.59\rangle
\end{array}\right.
$$

$\left.\begin{array}{ccccc}\langle 0.89,0.31,0.61\rangle & \langle 0.69,0.16,0.53\rangle & \langle 0.55,0.12,0.53\rangle & \langle 0.61,0.13,0.58\rangle & \langle 0.53,0.11,0.53\rangle \\ \langle 0.84,0.18,0.48\rangle & \langle 0.68,0.08,0.43\rangle & \langle 0.64,0.05,0.43\rangle & \langle 0.65,0.10,0.49\rangle & \langle 0.71,0.13,0.49\rangle \\ \langle 0.84,0.26,0.54\rangle & \langle 0.72,0.17,0.51\rangle & \langle 0.69,0.16,0.53\rangle & \langle 0.64,0.13,0.53\rangle & \langle 0.73,0.22,0.57\rangle \\ \langle 0.70,0.22,0.58\rangle & \langle 0.63,0.18,0.60\rangle & \langle 0.65,0.21,0.65\rangle & \langle 0.55,0.13,0.65\rangle & \langle 0.59,0.16,0.62\rangle \\ \langle 0.50,0,0.50\rangle & \langle 0.59,0.1,0.53\rangle & \langle 0.53,0.11,0.62\rangle & \langle 0.50,0.12,0.65\rangle & \langle 0.57,0.21,0.73\rangle \\ \langle 0.59,0.1,0.53\rangle & \langle 0.50,0,0.50\rangle & \langle 0.54,0.10,0.58\rangle & \langle 0.62,0.22,0.83\rangle & \langle 0.55,0.13,0.63\rangle \\ \langle 0.62,0.11,0.53\rangle & \langle 0.58,0.10,0.54\rangle & \langle 0.50,0,0.50\rangle & \langle 0.53,0.10,0.62\rangle & \langle 0.55,0.10,0.59\rangle \\ \langle 0.65,0.12,0.50\rangle & \langle 0.83,0.22,0.62\rangle & \langle 0.62,0.10,0.53\rangle & \langle 0.50,0,0.50\rangle & \langle 0.56,0.08,0.54\rangle \\ \langle 0.73,0.21,0.57\rangle & \langle 0.63,0.13,0.55\rangle & \langle 0.59,0.10,0.55\rangle & \langle 0.54,0.08,0.56\rangle & \langle 0.50,0,0.50\rangle\end{array}\right]$

\section{Box II}

all acceptably consistent and since the weight of six experts was $\left(\frac{1}{6}, \frac{1}{6}, \frac{1}{6}, \frac{1}{6}, \frac{1}{6}, \frac{1}{6}\right)^{T}$, the aggregated matrices (divided by seven attributes) could be computed. As an example, the aggregated preference values under attribute $C_{1}$ are shown in Box I (the original 42 matrices are omitted, which are transformed from the case study in [14]).
We then calculated the group decision matrix, based on seven matrices, in Box II. If the acceptable consistency threshold is $\theta=0.9$, then according to Eq. (10), the consistency index of $\tilde{A}$ becomes $C I(\tilde{A})=$ $0.99002>\theta=0.9$. Therefore, $\tilde{A}$ is an acceptably consistent NFPR. Then, $S\left(A_{i}\right)=\sum_{j=1}^{n} S\left(\tilde{a}_{i j}\right)$ is com- 
Table 1. Final scores and ranking of nine alternatives by NFPRs.

\begin{tabular}{ccc}
\hline $\begin{array}{c}\text { Medical tourism } \\
\text { sites }\end{array}$ & $\begin{array}{c}\text { Final score } \\
\left(\boldsymbol{S}_{\left.\left(\boldsymbol{A}_{\boldsymbol{i}}\right)\right)}\right.\end{array}$ & Ranking \\
\hline$A_{1}$ & 0.246 & 3 \\
$A_{2}$ & 1.523 & 1 \\
$A_{3}$ & 0.900 & 2 \\
$A_{4}$ & -0.499 & 7 \\
$A_{5}$ & -1.197 & 9 \\
$A_{6}$ & -0.690 & 8 \\
$A_{7}$ & -0.368 & 6 \\
$A_{8}$ & 0.221 & 4 \\
$A_{9}$ & -0.065 & 5 \\
\hline
\end{tabular}

puted in order to rank the sites (alternatives) based on Definition 3.4. For instance, we can get:

$$
\begin{aligned}
S\left(A_{1}\right)= & 0-0.116-0.070+0.098+0.166 \\
& +0.131+0.017+0.019+0.001=0.246, \\
S\left(A_{2}\right)= & 0.116+0+0.059+0.273+0.326 \\
& +0.230+0.207+0.144+0.169=1.523 .
\end{aligned}
$$

The ranking is therefore done (as shown in Table 1 ); the bigger the value of $S\left(A_{i}\right)$, the better is the destination. We concluded that $A_{2}$ (Chennai) was the best place for medical tourism in India.

In order to demonstrate the validity of MCGDMNFPRs, we compared MCGDM-NFPRs with rough AHP-MABAC 1. The comparison is listed in Table 2, from which it is clear that the two approaches have the same best alternative and similar rankings, which indicates the validity of our method.

Furthermore, because the original 42 matrices, the aggregated 7 matrices (under 7 attributes), and the final aggregated matrix were all adapted to satisfy consistency, the results of the proposed method were different to those of rough AHP-MABAC. For example, if the original 42 matrices did not accept normalization, the ranking would be $A_{2}>A_{3}>A_{1}>A_{8}>A_{9}>$ $A_{4}=A_{7}>A_{6}>A_{5}$. These rankings are depicted in Figure 2.

\subsection{Illustration of the proposed method (Case 2)}

The resource and processing of data have been described in Section 6.1. A group of four medical

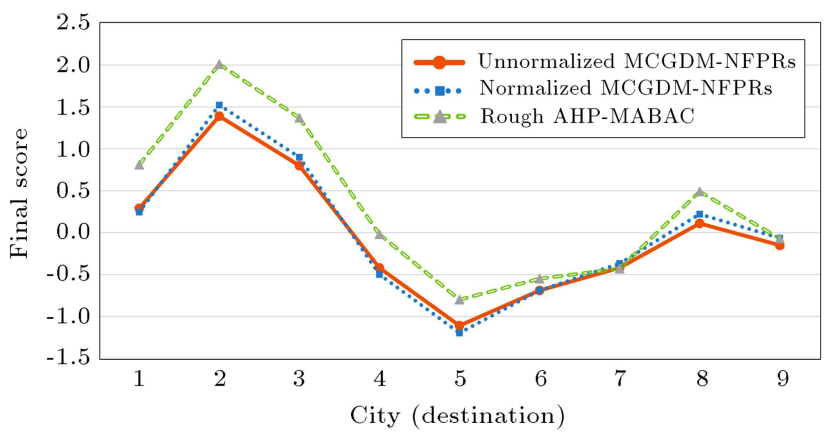

Figure 2. The final rankings of $x_{i}$ according to three approaches.

tourists represented as $D=\left\{d_{1}, d_{2}, d_{3}, d_{4}\right\}$ were asked to express their preference values based on their own knowledge and experience. They expressed their preference for one city (destination) over another one among a total of four cities $X=\left\{x_{1}, x_{2}, \ldots, x_{4}\right\}$ using NFPRs $\tilde{A}_{z}=\left(\tilde{a}_{z i j}\right)_{n \times n}$ with $\tilde{a}_{z i j}=\left\langle T_{\tilde{a}_{z i j}}, I_{\tilde{a}_{z i j}}, F_{\tilde{a}_{i j}}\right\rangle$, where $z \in\{1,2,3,4\}$ and $i, j \in\{1,2,3,4\}$. The original four matrices are shown in Box III. According to Eqs. (10)-(12), one can calculate the consistency level $C I\left(\tilde{A}_{z}\right)$ of every NFPR $\tilde{A}_{z}$ for $z=1,2,3,4$. The result is shown below:

$$
\begin{aligned}
& C I\left(\tilde{A}_{1}\right)=0.9583, \quad C I\left(\tilde{A}_{2}\right)=0.905 \\
& C I\left(\tilde{A}_{3}\right)=0.7583, \quad C I\left(\tilde{A}_{4}\right)=0.7167 .
\end{aligned}
$$

Let the acceptable consistency threshold be $\theta=$ 0.9 ; then, based on Definition 3.9, the NFPRs $\tilde{A}_{1}$ and $\tilde{A}_{2}$ are acceptably consistent. Therefore, $\tilde{A}_{1}(\lambda)=\tilde{A}_{1}$ and $\tilde{A}_{2}(\lambda)=\tilde{A}_{2}$.

On the other hand, because $C I\left(\tilde{A}_{3}\right)=0.7583<$ $\theta=0.9$ and $C I\left(\tilde{A}_{4}\right)=0.7167<\theta=0.9$, one can get the transformation of $\tilde{A}_{3}$ and $\tilde{A}_{4}$, represented as $\hat{A}_{3}$ and $\hat{A}_{4}$, respectively. As per Eq. (15), there exists $\hat{I}_{442}=\hat{I}_{424}=-0.034$ in matrix $\hat{A}_{4}$. Based on Eq. (16), $t=0.029$; accordingly, the rectified matrix $\tilde{A}_{3}^{\prime}=\left(\tilde{a}_{3 i j}^{\prime}\right)_{n \times n}=\hat{A}_{3}=\left(\hat{a}_{3 i j}\right)_{n \times n} \hat{A}_{4}^{\prime}=\left(\hat{a}_{4 i j}^{\prime}\right)_{n \times n}$ is constructed as shown in Box IV.

As per Eq. (17) and Theorem 4.4, we can get:

$$
\begin{array}{r}
\tilde{T}_{3 i j}(\lambda)\left(1-\tilde{I}_{3 i j}(\lambda)\right)=(1-\lambda) \tilde{T}_{3 i j}\left(1-\tilde{I}_{3 i j}\right) \\
+\lambda \tilde{T}_{3 i j}^{\prime}\left(1-\tilde{I}_{3 i j}^{\prime}\right)=0.414 \tilde{T}_{3 i j}\left(1-\tilde{I}_{3 i j}\right)
\end{array}
$$

Table 2. Comparison of two methods.

\begin{tabular}{cc}
\hline Methods & Ranking order \\
\hline Rough AHP-MABAC & $A_{2}>A_{3}>A_{1}>A_{8}>A_{4}>A_{9}>A_{7}>A_{6}>A_{5}$ \\
Proposed MCGDM-NFPRs & $A_{2}>A_{3}>A_{1}>A_{8}>A_{9}>A_{7}>A_{4}>A_{6}>A_{5}$ \\
\hline
\end{tabular}




$$
\begin{aligned}
\tilde{A}_{1}= & {\left[\begin{array}{cccc}
\langle 0.5,0,0.5\rangle & \langle 0.9,0.5,0.5\rangle & \langle 0.375,0.2,0.5\rangle & \langle 0.8,0.5,0.9\rangle \\
\langle 0.5,0.5,0.9\rangle & \langle 0.5,0,0.5\rangle & \langle 0.4,0.5,0.7\rangle & \langle 0.5,0.4,0.75\rangle \\
\langle 0.5,0.2,0.375\rangle & \langle 0.7,0.5,0.4\rangle & \langle 0.5,0,0.5\rangle & \langle 0.7,0.5,0.9\rangle \\
\langle 0.9,0.5,0.8\rangle & \langle 0.75,0.4,0.5\rangle & \langle 0.9,0.5,0.7\rangle & \langle 0.5,0,0.5\rangle
\end{array}\right] } \\
\tilde{A}_{2}= & {\left[\begin{array}{cccc}
\langle 0.5,0,0.5\rangle & \langle 0.2,0.1,0.4\rangle & \langle 0.35,0,0.3\rangle & \langle 0.45,0,0.25\rangle \\
\langle 0.4,0.1,0.2\rangle & \langle 0.5,0,0.5\rangle & \langle 0.65,0.2,0.25\rangle & \langle 0.35,0,0.45\rangle \\
\langle 0.3,0,0.35\rangle & \langle 0.25,0.2,0.65\rangle & \langle 0.5,0,0.5\rangle & \langle 0.3,0,0.45\rangle \\
\langle 0.25,0,0.45\rangle & \langle 0.45,0,0.35\rangle & \langle 0.45,0,0.3\rangle & \langle 0.5,0,0.5\rangle
\end{array}\right] } \\
\tilde{A}_{3}= & {\left[\begin{array}{cccc}
\langle 0.5,0,0.5\rangle & \langle 0.4,0,0.55\rangle & \langle 0.05,0,0.9\rangle & \langle 0.1,0,0.8\rangle \\
\langle 0.55,0,0.4\rangle & \langle 0.5,0,0.5\rangle & \langle 0.3,0,0.6\rangle & \langle 0.05,0,0.85\rangle \\
\langle 0.9,0,0.05\rangle & \langle 0.6,0,0.3\rangle & \langle 0.5,0,0.5\rangle & \langle 0.95,0,0\rangle \\
\langle 0.8,0,0.1\rangle & \langle 0.85,0,0.05\rangle & \langle 0,0,0.95\rangle & \langle 0.5,0,0.5\rangle
\end{array}\right] } \\
\tilde{A}_{4}= & {\left[\begin{array}{cccc}
\langle 0.5,0,0.5\rangle & \langle 0.1,0,0.7\rangle & \langle 0.8,0,0.1\rangle & \langle 0.5,0,0.4\rangle \\
\langle 0.7,0,0.1\rangle & \langle 0.5,0,0.5\rangle & \langle 0.7,0,0.1\rangle & \langle 0.3,0,0\rangle \\
\langle 0.1,0,0.8\rangle & \langle 0.1,0,0.7\rangle & \langle 0.5,0,0.5\rangle & \langle 0.8,0,0.1\rangle \\
\langle 0.4,0,0.5\rangle & \langle 0,0,0.3\rangle & \langle 0.1,0,0.8\rangle & \langle 0.5,0,0.5\rangle
\end{array}\right] }
\end{aligned}
$$

Box III

$$
\begin{array}{r}
\tilde{A}_{3}^{\prime}=\left[\begin{array}{cccc}
\langle 0.5,0,0.5\rangle & \langle 0.5278,0.1136,0.55\rangle & \langle 0.2275,0.1574,0.9\rangle & \langle 0.4255,0.2656,0.8\rangle \\
\langle 0.55,0.1136,0.5218\rangle & \langle 0.5,0,0.5\rangle & \langle 0.1792,0,0.7208\rangle & \langle 0.4804,0.3235,0.85\rangle \\
\langle 0.9,0.1574,0.2275\rangle & \langle 0.7208,0,0.1792\rangle & \langle 0.5,0,0.5\rangle & \langle 0.6208,0,0.3292\rangle \\
\langle 0.8,0.2656,0.4255\rangle & \langle 0.85,0.3235,0.4804\rangle & \langle 0.3292,0,0.6208\rangle & \langle 0.5,0,0.5\rangle
\end{array}\right] \\
\tilde{A}_{4}^{\prime}=\left[\begin{array}{cccc}
\langle 0.5,0,0.5\rangle & \langle 0.5761,0.3644,0.7\rangle & \langle 0.5276,0,0.3780\rangle & \langle 0.7219,0.1929,0.4\rangle \\
\langle 0.7,0.3644,0.5761\rangle & \langle 0.5,0,0.5\rangle & \langle 0.5197,0,0.2913\rangle & \langle 0.3386,0,0\rangle \\
\langle 0.3780,0,0.5276\rangle & \langle 0.2913,0,0.5197\rangle & \langle 0.5,0,0.5\rangle & \langle 0.5078,0,0.3976\rangle \\
\langle 0.4,0.1929,0.7219\rangle & \langle 0,0,0.3386\rangle & \langle 0.3976,0,0.5079\rangle & \langle 0.5,0,0.5\rangle
\end{array}\right]
\end{array}
$$

\section{Box IV}

$$
+0.586 \tilde{T}_{3 i j}^{\prime}\left(1-\tilde{I}_{3 i j}^{\prime}\right)
$$

and:

$$
\begin{aligned}
& \tilde{T}_{3 i j}(\lambda)\left(1-\tilde{I}_{3 i j}(\lambda)\right)=(1-\lambda) \tilde{T}_{3 i j}\left(1-\tilde{I}_{3 i j}\right) \\
& +\lambda \tilde{T}_{3 i j}^{\prime}\left(1-\tilde{I}_{3 i j}^{\prime}\right)=0.353 \tilde{T}_{3 i j}\left(1-\tilde{I}_{3 i j}\right) \\
& \quad+0.647 \tilde{T}_{3 i j}^{\prime}\left(1-\tilde{I}_{3 i j}^{\prime}\right)
\end{aligned}
$$

Therefore, the weighted averaging matrices $\tilde{A}_{3}(\lambda)$ and $\tilde{A}_{4}(\lambda)$ are obtained as shown in Box V. The two NFPRs $\tilde{A}_{3}(\lambda)$ and $\tilde{A}_{4}(\lambda)$ are acceptably consistent with $C I\left(\tilde{A}_{3}(\lambda)\right)=0.9$ and $C I\left(\tilde{A}_{4}(\lambda)\right)=0.9$. The MAD values between $\tilde{A}_{z}$ and $\tilde{A}_{z}(\lambda)$, where $z \in$ $\{1,2,3,4\}$, are then computed as follows:

$$
\begin{aligned}
& \operatorname{MAD}\left(\tilde{A}_{1}(\lambda), \tilde{A}_{1}\right)=0 \\
& \operatorname{MAD}\left(\tilde{A}_{2}(\lambda), \tilde{A}_{2}\right)=0 \\
& \operatorname{MAD}\left(\tilde{A}_{3}(\lambda), \tilde{A}_{3}\right)=0.11154, \\
& M A D\left(\tilde{A}_{4}(\lambda), \tilde{A}_{4}\right)=0.12033 .
\end{aligned}
$$

The value of $H_{z}$ for any $z \in\{1,2,3,4\}$ can be obtained by plugging $M A D\left(\tilde{A}_{z}(\lambda), \tilde{A}_{z}\right)$ and $C I\left(\tilde{A}_{z}(\lambda)\right)$ : $H_{1}=0.9792, H_{2}=0.9525, H_{3}=0.8942, H_{4}=0.8898$. Based on the value of $H_{z}$ and Eq. (22), the associated weight vectors, $W=\left(w_{1}, w_{2}, w_{3}, w_{4}\right)^{T}$, were calculated as shown in Table 3 and Figure 3.

We can see in Table 3 and Figure 3 that the greater the value of $\beta$, the smaller the sum of the 


\begin{aligned} \hline$\tilde{A}_{3}(\lambda)=\left[\begin{array}{cccc}\langle 0.5,0,0.5\rangle & \langle 0.4678,0.0666,0.55\rangle & \langle 0.1466,0.0923,0.9\rangle & \langle 0.2660,0.1557,0.8\rangle \\ \langle 0.55,0.0666,0.4678\rangle & \langle 0.5,0,0.5\rangle & \langle 0.2292,0,0.6708\rangle & \langle 0.2606,0.1897,0.85\rangle \\ \langle 0.9,0.0923,0.1466\rangle & \langle 0.6708,0,0.2292\rangle & \langle 0.5,0,0.5\rangle & \langle 0.7570,0,0.1930\rangle \\ \langle 0.8,0.1557,0.2660\rangle & \langle 0.85,0.1897,0.2606\rangle & \langle 0.1930,0,0.7570\rangle & \langle 0.5,0,0.5\rangle\end{array}\right] \\ \tilde{A}_{4}(\lambda)=\left[\begin{array}{cccc}\langle 0.5,0,0.5\rangle & \langle 0.3562,0.2358,0.7\rangle & \langle 0.6237,0,0.2799\rangle & \langle 0.6324,0.1248,0.4\rangle \\ \langle 0.7,0.2358,0.3562\rangle & \langle 0.5,0,0.5\rangle & \langle 0.5833,0,0.2238\rangle & \langle 0.3250,0,0\rangle \\ \langle 0.2799,0,0.6237\rangle & \langle 0.2238,0,0.5833\rangle & \langle 0.5,0,0.5\rangle & \langle 0.6110,0,0.2926\rangle \\ \langle 0.4,0.1248,0.6324\rangle & \langle 0,0,0.3250\rangle & \langle 0.2926,0,0.6110\rangle & \langle 0.5,0,0.5\rangle\end{array}\right]\end{aligned}$

Box V

Table 3. The values of associated weights according to different values of $\beta$.

\begin{tabular}{ccccccccc}
\hline $\boldsymbol{\beta}$ & $\boldsymbol{w}_{\mathbf{1}}$ & $\boldsymbol{w}_{\boldsymbol{2}}$ & $\boldsymbol{w}_{\boldsymbol{3}}$ & $\boldsymbol{w}_{\boldsymbol{4}}$ & $\boldsymbol{w}_{\mathbf{1}}-\boldsymbol{w}_{\mathbf{2}}$ & $\boldsymbol{w}_{\boldsymbol{2}}-\boldsymbol{w}_{\boldsymbol{3}}$ & $\boldsymbol{w}_{\boldsymbol{3}}-\boldsymbol{w}_{\mathbf{4}}$ & $\sum_{\boldsymbol{i}=\mathbf{1}}^{\mathbf{3}}\left(\boldsymbol{w}_{\boldsymbol{i}}-\boldsymbol{w}_{\boldsymbol{i}+\mathbf{1}}\right)$ \\
\hline 0 & 1 & 0 & 0 & 0 & 1 & 0 & 0 & 1 \\
0.1 & 0.875 & 0.062 & 0.036 & 0.027 & 0.814 & 0.025 & 0.009 & 0.848 \\
0.2 & 0.766 & 0.111 & 0.069 & 0.053 & 0.654 & 0.042 & 0.016 & 0.713 \\
0.3 & 0.670 & 0.152 & 0.099 & 0.079 & 0.519 & 0.052 & 0.021 & 0.591 \\
0.4 & 0.587 & 0.183 & 0.127 & 0.104 & 0.403 & 0.057 & 0.023 & 0.483 \\
0.5 & 0.513 & 0.208 & 0.151 & 0.128 & 0.306 & 0.057 & 0.023 & 0.385 \\
0.6 & 0.449 & 0.226 & 0.173 & 0.151 & 0.223 & 0.053 & 0.022 & 0.298 \\
0.7 & 0.393 & 0.239 & 0.193 & 0.174 & 0.154 & 0.046 & 0.019 & 0.219 \\
0.8 & 0.344 & 0.248 & 0.211 & 0.197 & 0.096 & 0.038 & 0.014 & 0.147 \\
0.9 & 0.301 & 0.254 & 0.227 & 0.218 & 0.047 & 0.027 & 0.008 & 0.083 \\
1 & 0.264 & 0.256 & 0.241 & 0.239 & 0.007 & 0.016 & 0.001 & 0.024 \\
\hline
\end{tabular}

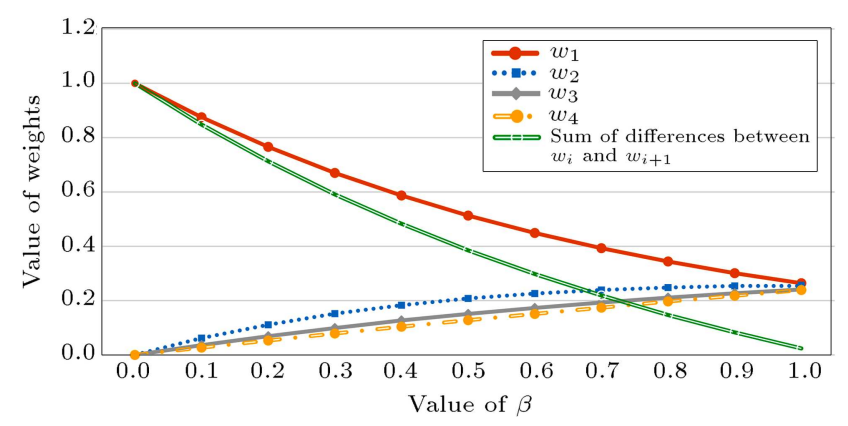

Figure 3. The values of associated weights based on different $\beta$ values.

differences between the two values of weights $w_{i}$ and $w_{i+1}$, where $i=1,2,3$. In this example, we let $\beta=1$; then, the weights are $W=(0.264,0.256,0.241,0.239)^{T}$. One can get the aggregated NFPR $\tilde{G}=\left(\tilde{g}_{i j}\right)_{n \times n}$ on the basis of $W$ and permutation $\sigma$, as written in Box VI. As per Eq. (10), the consistency index of $\tilde{G}$ is
$C I(\tilde{G})=0.96006>\theta=0.9$, so the aggregated NFPR $\tilde{G}$ is acceptably consistent.

According to Definition 3.4, $S\left(\tilde{g}_{i}\right)=\sum_{j=1}^{n} S\left(\tilde{g}_{i j}\right)$ was calculated to rank the cities. We first obtained the individual values $S\left(\tilde{g}_{1}\right)=-0.1855, S\left(\tilde{g}_{2}\right)=-0.0924$, $S\left(\tilde{g}_{3}\right)=0.2124$, and $S\left(\tilde{g}_{4}\right)=0.0655$ followed by $S\left(\tilde{g}_{3}\right)>S\left(\tilde{g}_{4}\right)>S\left(\tilde{g}_{2}\right)>S\left(\tilde{g}_{1}\right)$; therefore, the ranking of four cities was $x_{3} \succ x_{4} \succ x_{2} \succ x_{1}$. In order to analyze the parameter $\beta$, we computed the results of ranking as listed in Table 4 and Figure 4.

The rankings based on $\tilde{A}_{z}(\lambda)$ with $z \in\{1,2,3,4\}$, which indicate the original preferences of four DMs (tourists), are stated as follows.

\subsection{Discussions}

In this section, we discuss the proposed MCGDMNFPRs in comparison with the other two methods, accordingto Tables 1-5 and Figures 1-6 and based on

$$
\tilde{G}=\left[\begin{array}{cccc}
\langle 0.5,0,0.5\rangle & \langle 0.486,0.230,0.534\rangle & \langle 0.373,0.075,0.492\rangle & \langle 0.542,0.199,0.590\rangle \\
\langle 0.534,0.230,0.486\rangle & \langle 0.5,0,0.5\rangle & \langle 0.467,0.183,0.464\rangle & \langle 0.362,0.151,0.518\rangle \\
\langle 0.492,0.075,0.373\rangle & \langle 0.464,0.183,0.467\rangle & \langle 0.5,0,0.5\rangle & \langle 0.590,0.132,0.469\rangle \\
\langle 0.590,0.199,0.542\rangle & \langle 0.518,0.151,0.362\rangle & \langle 0.469,0.132,0.590\rangle & \langle 0.5,0,0.5\rangle
\end{array}\right]
$$


Table 4. The rankings of $x_{i}$ based on different $\beta$ values.

\begin{tabular}{cccccc}
\hline $\boldsymbol{\beta}$ & $\boldsymbol{S}\left(\tilde{\boldsymbol{g}}_{\mathbf{1}}\right)$ & $\boldsymbol{S}\left(\tilde{\boldsymbol{g}}_{\mathbf{2}}\right)$ & $\boldsymbol{S}\left(\tilde{\boldsymbol{g}}_{\mathbf{3}}\right)$ & $\boldsymbol{S}\left(\tilde{\boldsymbol{g}}_{\mathbf{4}}\right)$ & Ranking of $\boldsymbol{x}_{\boldsymbol{i}}$ \\
\hline 0 & 0.05 & -0.5 & 0.15 & 0.3 & $x_{4} \succ x_{3} \succ x_{1} \succ x_{2}$ \\
0.1 & 0.0271 & -0.4653 & 0.1493 & 0.2889 & $x_{4} \succ x_{3} \succ x_{1} \succ x_{2}$ \\
0.2 & 0.0015 & -0.4240 & 0.1510 & 0.2715 & $x_{4} \succ x_{3} \succ x_{1} \succ x_{2}$ \\
0.3 & -0.0254 & -0.3792 & 0.1549 & 0.2498 & $x_{4} \succ x_{3} \succ x_{1} \succ x_{2}$ \\
0.4 & -0.0524 & -0.3335 & 0.1606 & 0.2253 & $x_{4} \succ x_{3} \succ x_{1} \succ x_{2}$ \\
0.5 & -0.0787 & -0.2882 & 0.1677 & 0.1992 & $x_{4} \succ x_{3} \succ x_{1} \succ x_{2}$ \\
0.6 & -0.1037 & -0.2444 & 0.1758 & 0.1722 & $x_{3} \succ x_{4} \succ x_{1} \succ x_{2}$ \\
0.7 & -0.1270 & -0.2027 & 0.1847 & 0.1451 & $x_{3} \succ x_{4} \succ x_{1} \succ x_{2}$ \\
0.8 & -0.1485 & -0.1634 & 0.1939 & 0.1181 & $x_{3} \succ x_{4} \succ x_{1} \succ x_{2}$ \\
0.9 & -0.1680 & -0.1267 & 0.2032 & 0.0915 & $x_{3} \succ x_{4} \succ x_{2} \succ x_{1}$ \\
1 & -0.1855 & -0.0924 & 0.2124 & 0.0655 & $x_{3} \succ x_{4} \succ x_{2} \succ x_{1}$ \\
\hline
\end{tabular}

Table 5. The rankings of $x_{i}$ for $\tilde{A}_{z}(\lambda)$.

\begin{tabular}{cccccc}
\hline & $\boldsymbol{S}_{\mathbf{1}}$ & $\boldsymbol{S}_{\mathbf{2}}$ & $\boldsymbol{S}_{\mathbf{3}}$ & $\boldsymbol{S}_{\mathbf{4}}$ & Ranking of $\boldsymbol{x}_{\boldsymbol{i}}$ \\
\hline$\tilde{A}_{1}(\lambda)$ & 0.05 & -0.5 & 0.15 & 0.3 & $x_{4} \succ x_{3} \succ x_{1} \succ x_{2}$ \\
$\tilde{A}_{2}(\lambda)$ & 0.07 & 0.4 & -0.52 & 0.05 & $x_{2} \succ x_{1} \succ x_{4} \succ x_{3}$ \\
$\tilde{A}_{3}(\lambda)$ & -1.21 & -0.84 & 1.68 & 0.36 & $x_{3} \succ x_{4} \succ x_{2} \succ x_{1}$ \\
$\tilde{A}_{4}(\lambda)$ & 0.28 & 0.94 & -0.38 & -0.85 & $x_{2} \succ x_{1} \succ x_{3} \succ x_{4}$ \\
\hline
\end{tabular}

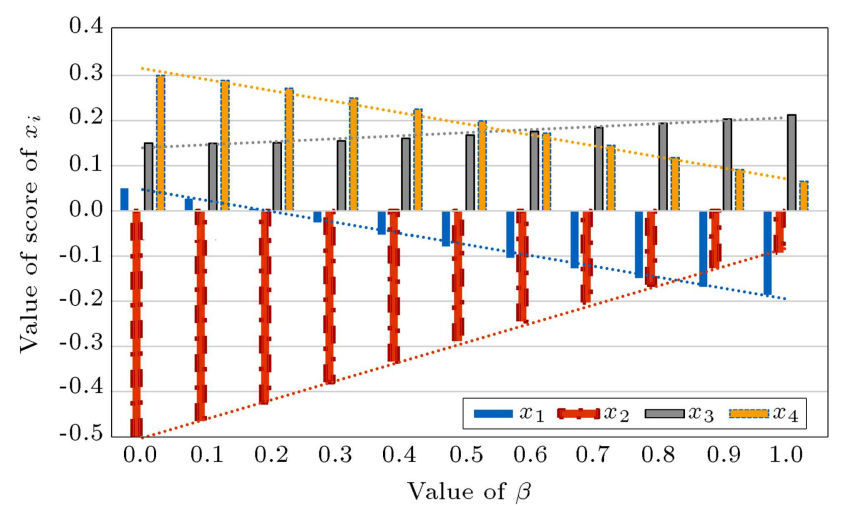

Figure 4. The rankings of $x_{i}$ according to different $\beta$ values.

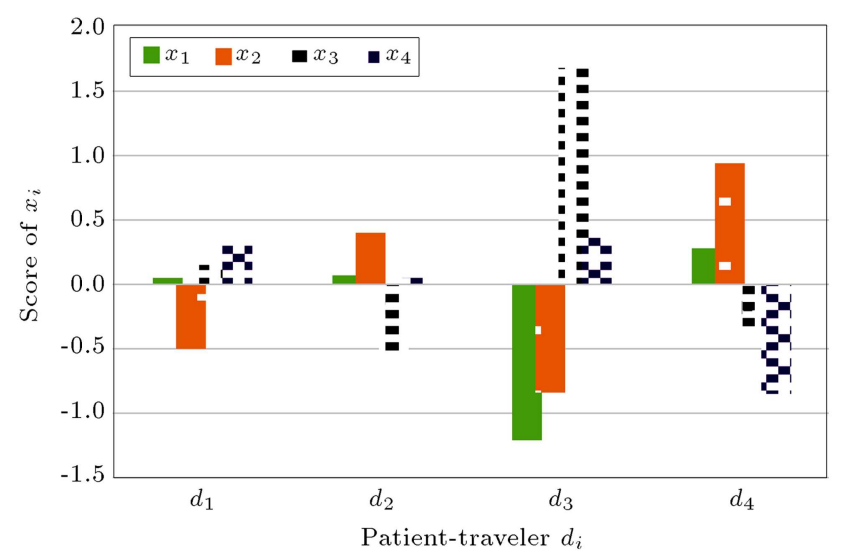

Figure 5. The original ranking of $x_{i}$ for the four patient-travelers $D=\left\{d_{1}, d_{2}, d_{3}, d_{4}\right\}$.

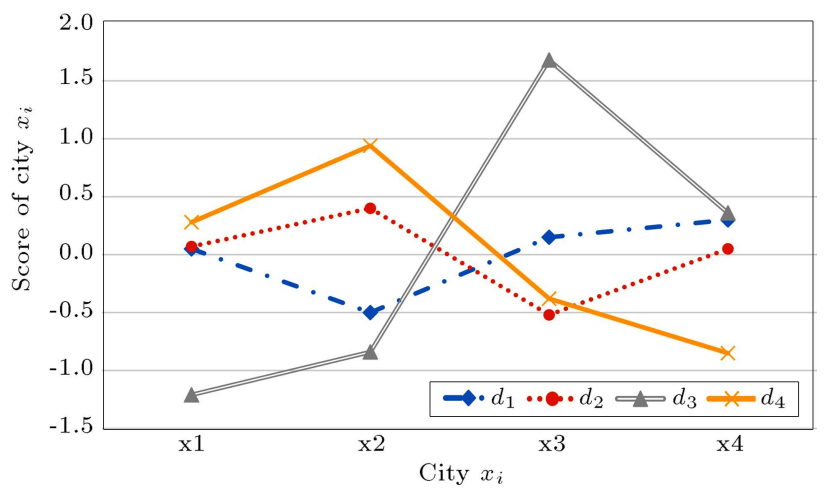

Figure 6. The original ranking of $x_{i}$ for every traveler.

Cases 1, and 2. The findings are discussed in the following six parts:

(a) As shown in Table 1 and Figure 1, the results for the best destination are the same in our proposed approach and rough AHP-MABAC. It is also clear from Figure 1 that the tendencies of rankings of the two methods are similar, hence validating our proposed method, especially the usefulness of the proposed MAD based aggregation approach;

(b) Table 2 shows that the rankings of the cities (alternatives) of Hyderabad $\left(A_{4}\right)$ and Kochi $\left(A_{9}\right)$ are different in two methods for Case 1. In order to find out the reason, we displayed the ranking of our method using the un-normalized original data in Figure 1 and observed that the rankings of $A_{4}$ and $A_{9}$ in the un-normalized data were closer to the ranking of rough AHP-MABAC than to that of normalized data. However, since the normalized data were logically consistent, they helped in avoiding inconsistent information during the decision making. Therefore, our proposed method could help in deducing any information inconsistency or distortion in the given information;

(c) The preference value for one alternative over another one would be fixed, no matter how the other alternatives change; thus, our method would not change the best alternative when a non-optimal alternative is replaced by another worse alternative, further proving the stability of MCGDM-NFPRs;

(d) From Table 4 and Figure 4, we could see that the ranking of $x_{i}$ changed with $\beta$. Since the weights of $\tilde{A}_{1}(\lambda)$ and $\tilde{A}_{3}(\lambda)$ are always higher than those of $\tilde{A}_{2}(\lambda)$ and $\tilde{A}_{4}(\lambda)$, respectively, there should exist $x_{4}, x_{3} \succ x_{1}, x_{2}$. When $0 \leq \beta \leq 0.5$, the differences between the weights of $w_{1}, w_{2}$ and $w_{3}, w_{4}$ are significant, so $x_{4} \succ x_{3} ; x_{3} \succ x_{4}$; while $\beta \geq 0.5$. On the other hand, when $\beta \leq 0.8$, the weight $w_{1}$ is significant so that $x_{1} \succ x_{2}$ and $x_{2} \succ x_{1}$ when $\beta>0.8$. We can then draw the 
conclusion that if the DMs wish to differentiate the experts' judgments, not to make the difference too big, a greater value of $\beta$ will be more appropriate;

(e) According to Table 5 and Figures 5 and 6, the original rankings of the four alternatives (enterprises) are different for the four experts (executives), which implies that the preferences of four experts differ; this is a common phenomenon and a crucial problem in the process of GDM. Our algorithm can deal with this issue with NFPRs that are more flexible and applicable than the IPPRs, since they help DMs express their preferences comprehensively and in more detail. Furthermore, the ranking in the result of the example in which is the same as that of Wang et al. [29], which indicated the feasibility of our proposed method;

(f) In Case 2, the results of the proposed method and Wang et al. [29] are the same. However, this paper uses NFPRs instead of the IPRs in [29]; therefore, it can express more information. In addition, we can set the indeterminacy-membership function of an SVNS to zero, instead of an IFS, making our method more flexible.

\section{Conclusion}

At present, the medical tourism industry is booming around the world, due to the economic prosperity, cultural development, and frequent exchanges between countries. However, a vital problem for a patienttourist is to choose the ideal city based on the infrastructure, services, and policies. This paper proposed the MCGDM-NFPRs model and applied it to the evaluation and selection of the optimum medical tourism destinations. The MCGDM-NFPRs model under neutrosophic environment was mainly composed of four aspects: (1) the measurement of additive consistency and acceptable consistency for NFPRs, (2) the approach to improve the consistency of unacceptably consistent NFPRs, (3) the aggregation method for NFPRs, and (4) the way to rank the alternatives represented by the NFPRs.

Two illustrative examples were put up to verify the practicability and validity of the proposed approach by comparing it with other two methods. The results clearly indicated that the proposed approach was a valid, stable, and convenient tool to evaluate and prioritize healthcare tourism destinations.

Further work is necessary to solve the original incomplete data, describing the preference values among alternatives (cities). The main obstacles are how to fill up the missing data according to the logic of experts and the universality of SVNEs, and how to compute the consistency index of incomplete NFPR accurately. Another topic that needs to be considered is how to recommend different destinations for different people, i.e., personalized or 'tailor-made' recommendations.

\section{Acknowledgments}

This work was partially supported by the National Natural Science Foundation of China (Grant Nos. 71871229, 71771219). The constructive suggestions from the anonymous referees are gratefully acknowledged.

\section{References}

1. Connell, J. "Medical tourism: Sea, sun, sand and surgery", Tour. Mgt., 27(6), pp. 1093-1100 (2006). DOI:10.1016/j.tourman.2005.11.005

2. Rosensweig, J.A. "Medical tourism-health care in the global economy", Physician Executive, 33(6), pp. 2830 (2007).

3. Horowitz, M.D., Rosensweig, J.A., and Jones, C.A. "Medical tourism: globalization of the healthcare marketplace", Medscape General Medicine, 9(4), p. 33 (2007).

4. Crush, J. and Chikanda, A. "South-South medical tourism and the quest for health in Southern Africa", Soc. Sci. Med., 124, pp. 313-320 (2015). DOI: 10.1016/j.socscimed.2014.06.025

5. Thinakorn, N., Johanna, H., and Richard, S. "Medical tourism in Thailand: a cross-sectional study", Bull. World Health Organ., 94(1), pp. 30-36 (2016). DOI: 10.2471/BLT.14.152165

6. Wongkit, M. and Mckercher, B. "Desired attributes of medical treatment and medical service providers: a case study of medical tourism in Thailand", J. Travel Tour. Mark., 33(1), pp. 1-14 (2015). DOI:10.1080/10548408.2015.1024911

7. Snyder, J., Byambaa, T., Johnston, R., Crooks, V.A., Janes, C., and Ewan, M. "Outbound medical tourism from Mongolia: a qualitative examination of proposed domestic health system and policy responses to this trend", BMC Health Serv. Res., 15(1), pp. 1-8 (2014). DOI:10.1186/s12913-015-0849-5

8. Cheng, K.M. "Medical tourism: Chinese maternity tourism to Hong Kong", Curr. Issues Tour., 19(14), pp. 1479-1486 (2015).

DOI: $10.1080 / 13683500.2015 .1042359$

9. Johnston, R., Crooks, V.A., Snyder, J., and Whitmore, R. "The major forces that need to back medical tourism were - in alignment: championing development of Barbados's medical tourism sector", Int. J. Health Serv., 45(2), pp. 334-352 (2015). DOI: $10.1177 / 0020731414568512$

10. Crooks, V.A., Turner, L., Snyder, J., Johnston, R., and Kingsbury, P. "Promoting medical tourism to India: Messages, images, and the marketing of international 
patient travel", Soc. Sci. Med., 72(5), pp. 726-732 (2011). DOI: $10.1016 /$ j.socscimed.2010.12.022

11. Junio, M.M.V., Kim, J.H., and Lee, T.J. "Competitiveness attributes of a medical tourism destination: The case of South Korea with importanceperformance analysis", 34(4), pp. 444-460 (2017). DOI: $10.1080 / 10548408.2016 .1182454$

12. Thornton, G. "Indian medical tourism industry to touch $\$ 8$ billion by $2020 "$ ", In The Economic Times (2015). https://economictimes.indiatimes.com/ industry/healthcare/biotech/healthcare/indianmedical-tourism-industry-to-touch-8-billion-by-2020grant-thornton/articleshow/49615898.cms.

13. Sultana, S., Haque, A., Momen, A., and Yasmin, F. "Factors affecting the attractiveness of medical tourism destination: An empirical study on Indiareview article", Iran J. Public Health, 43(7), pp. 867876 (2014).

14. Roy, J., Chatterjee, K., Bandhopadhyay, A., and Kar, S. "Evaluation and selection of medical tourism sites: A rough AHP based MABAC approach", arXiv: 1606.08962 (2016).

15. Eissler, L.A. and Casken, J. "Seeking health care through international medical tourism", Journal of Nursing Scholarship, 45(2), pp. 177-184 (2013).

16. Blin, J.M. "Fuzzy relations in group decision theory", J. Cybern., 4(2), pp. 17-22 (1974).

DOI: $10.1080 / 01969727408546063$

17. Herrera, F., Herrera Viedma, E., and Chiclana, F. "Multiperson decision-making based on multiplicative preference relations", Eur. J. Oper. Res., 129(2), pp. 372-385 (2001). DOI: 10.1016/S0377-2217(99)00197-6

18. Chiclana, F., Herrera, F., and Herrera Viedma, E. "Integrating multiplicative preference relations in a multipurpose decision-making model based on fuzzy preference relations", Fuzzy Sets Syst., 122(2), pp. 277-291 (2001). DOI: 10.1016/S0165-0114(00)00004-X.

19. Yu, D. and Li, D.F. "Managing interval-valued multiplicative hesitant fuzzy information in GDM problems", Sci. Iranica. Trans. E, Ind. Eng., 23(4), pp. 1918-1927 (2016).

20. Khanzadi, M., Nasirzadeh, F., Hassani, S.H., and Mohtashemi, N.N. "An integrated fuzzy multi-criteria group decision making approach for project delivery system selection", Sci. Iran. Trans. A, Civ. Eng., 23(3), pp. 802-814 (2016).

21. Atanassov, K.T. "Intuitionistic fuzzy sets", Fuzzy Sets Syst., 20(1), pp. 87-96 (1986). DOI: 10.1016/S01650114(86)80034-3

22. Wang, Z. and Li, K.W. "Group decision making with incomplete intuitionistic preference relations based on quadratic programming models", Comput. Ind. Eng., 93, pp. 162-170 (2016). DOI: 10.1016/j.cie.2016.01.001

23. Liu, H., Xu, Z., and Liao, H. "The multiplicative consistency index of hesitant fuzzy preference relation",
IEEE Trans. Fuzzy Syst., 24(1), pp. 82-93 (2016). DOI: 10.1109/TFUZZ.2015.2426315

24. Liao, H.C. and $\mathrm{Xu}$, Z.S. "Consistency of the fused intuitionistic fuzzy preference relation in group intuitionistic fuzzy analytic hierarchy process", Appl. Soft. Comput., 35, pp. 812-826 (2015). DOI:10.1016/j.asoc.2015.04.015

25. Wang, Z.J. and Li, K.W. "A multi-step goal programming approach for group decision making with incomplete interval additive reciprocal comparison matrices", Eur. J. Oper. Res., 242(3), pp. 890-900 (2015).

26. Chiclana, F., Ureña, R., Fujita, H., and HerreraViedma, E., Estimating Unknown Values in Reciprocal Intuitionistic Preference Relations via Asymmetric Fuzzy Preference Relations, pp. 66-77, Springer, Berlin (2015).

27. Ureña, R., Chiclana, F., Fujita, H., and Herrera Viedma, E. "Confidence-consistency driven group decision making approach with incomplete reciprocal intuitionistic preference relations", KnowledgeBased Syst., $\mathbf{8 9 ( C ) ,}$ pp. $86-96$ (2015). DOI: 10.1016/j.knosys.2015.06.020

28. Xu, Z. "Compatibility analysis of intuitionistic fuzzy preference relations in group decision making", Group Decis. Negot., 22(3), pp. 463-482 (2013). DOI: 10.1007/s10726-011-9278-y

29. Wang, Z.J., Wang, Y.H., and Li, K.W. "An acceptable consistency-based framework for group Decision Making with Intuitionistic Preference Relations", Group Decis. Negot., pp. 1-22 (2015). DOI: 10.1007/s10726015-9438-6

30. Wu, J. "Consistency in MCGDM problems with intuitionistic fuzzy preference relations based on an exponential score function", Group Decis. Negot., 25(2), pp. 399-420 (2016). DOI: 10.1007/s10726-015-9447-5

31. Zeng, S., Palacios Marques, D., and Zhu, F. "A new model for interactive group decision making with intuitionistic fuzzy preference relations", Informatica, 27(4), pp. 911-928 (2016).

32. Zhang, Y.B., Li, K.W., and Wang, Z.J. "Prioritization and aggregation of intuitionistic preference relations: A multiplicative-transitivity-based transformation from intuitionistic judgment data to priority weights", Group Decis. Negot., 26(2), pp. 409-436 (2017).

33. $\mathrm{Xu}, \mathrm{Z}$. and Cai, X. "Group decision making with incomplete interval-valued intuitionistic preference relations", Group Decis. Negot., 24(2), pp. 193-215 (2014). DOI: 10.1007/s10726-014-9386-6

34. Jiang, Y., Xu, Z., and Yu, X. "Group decision making based on incomplete intuitionistic multiplicative preference relations", Inf. Sci., 295, pp. 33-52 (2015). DOI: 10.1016/j.ins.2014.09.043

35. Zhou, W., Xu, Z., and Chen, M. "Preference relations based on hesitant-intuitionistic fuzzy information and their application in group decision making", 
Comput. Ind. Eng., 87, pp. 163-175 (2015). DOI: 10.1016/j.cie.2015.04.020

36. Wang, Z.J. and Tong, X.Y. "Consistency analysis and group decision making based on triangular fuzzy additive reciprocal preference relations", Inf. Sci., 361362(C), pp. 29-47 (2016).

37. Wang, Z.J. "Comments on "A note on "Applying fuzzy linguistic preference relations to the improvement of consistency of fuzzy AHP", Inf. Sci., 372, pp. 539-545 (2016).

38. Wang, Z.J. "Geometric consistency based interval weight elicitation from intuitionistic preference relations using logarithmic least square optimization", Fuuz. Optim. Decis. Mak., 14(3), pp. 289-310 (2015).

39. Wang, Z.J. and Zhang, X.Y. "A two-stage acceptable hesitancy based goal programming framework to evaluating missing values of incomplete intuitionistic reciprocal preference relations", Comput. Ind. Eng., 105, pp. 190-200 (2017).

40. Dubois, D.J., Fuzzy Sets and Systems: Theory and Applications, Academic Press (1980).

41. Zimmermann, H.-J., Fuzzy Set Theory-and Its Applications, Springer Science \& Business Media (2011).

42. Tanino, T. "Fuzzy preference relations in group decision making", In Non-conventional Preference Relations in Decision Making, pp. 54-71, Springer, Berlin Heidelberg (1988).

43. Tanino, T. "Fuzzy preference orderings in group decision making", Fuzzy Sets Syst., 12(2), pp. 117-131 (1984). DOI: $10.1016 / 0165-0114(84) 90032-0$

44. Liao, H., Xu, Z.S., and Xia, M.M. "Multiplicative consistency of hesitant fuzzy reference relation and its application in group decision making", Int. J. Inf. Technol. Decis. Mak., 13(1), pp. 47-76 (2014). DOI: 10.1142/S0219622014500035

45. Wang, H. and $\mathrm{Xu}, \mathrm{Z}$. "Some consistency measures of extended hesitant fuzzy linguistic preference relations", Inf. Sci., 297, pp. 316-331 (2015). DOI: 10.1016/j.ins.2014.10.047

46. Rallabandi, L.N.P.K., Vandrangi, R., and Rachakonda, S.R. "Improved consistency ratio for pairwise comparison matrix in analytic hierarchy processes", Asia. Pac. J. Oper. Res., 33, pp. 1650020-1650038 (2016).

47. Li, K.W., Wang, Z.J., and Tong, X.Y. "Acceptability analysis and priority weight elicitation for interval multiplicative comparison matrices", Eur. J. Oper. Res., 250(2), pp. 628-638 (2016).

48. Wang, Z.J. and Lin, J. "Acceptability measurement and priority weight elicitation of triangular fuzzy multiplicative preference relations based on geometric consistency and uncertainty indices", Inf. Sci., 402, pp. 105-123 (2017).

49. Wang, Z.J. "A two-stage linear goal programming approach to eliciting interval weights from additive interval fuzzy preference relations", Soft Computing, 20(7), pp. 2721-2732 (2016).

50. Smarandache, F. "A unifying field in logics: Neutrosophic logic. Neutrosophy, neutrosophic set, neutrosophic probability and statistics", (fourth edition), Journal of Molecular Biology, 332(2), pp. 489-503 (2003). DOI: $10.5281 /$ zenodo.8792

51. Wang, H., Smarandache, F., Zhang, Y., and Sunderraman, R. "Single valued neutrosophic sets", Tech. Sci. Appl. Math., 17(1), pp. 10-14 (2010).

52. Orlovsky, S. "Decision-making with a fuzzy preference relation", Fuzzy Sets Syst., 1(3), pp. 155-167 (1978). DOI: $10.1016 / 0165-0114(78) 90001-5$

53. Xu, Z. "Intuitionistic preference relations and their application in group decision making", Inf. Sci., 177(11), pp. $2363-2379$ (2007). DOI: 10.1016/j.ins.2006.12.019

54. Wang, Z.J. "Derivation of intuitionistic fuzzy weights based on intuitionistic fuzzy preference relations", Appl. Math. Model., 37(9), pp. 6377-6388 (2013). DOI: 10.1016/j.apm.2013.01.021

55. Wang, Z., Li, K.W., and Wang, W. "An approach to multiattribute decision making with interval-valued intuitionistic fuzzy assessments and incomplete weights", Information Science, 179(17), pp. 3026-3040 (2009).

56. Hu, J.H., Yang, Y., and Chen, X.H. "A novel TODIM method-based three-way decision model for medical treatment selection", International Journal of Fuzzy Systems, 20(4), pp. 1240-1255 (2018). DOI: $10.1007 / \mathrm{s} 40815-017-0320-3$

57. Wang, N.N. and Zhang, H.Y. "Probability multivalued linguistic neutrosophic sets for multi-criteria group decision-making", Int. J. Uncertain. Quantif., 7(3), pp. 207-228 (2017).

DOI: $10.1615 /$ Int.J.UncertaintyQuantification. 2017019632

58. Tian, Z.P., Wang, J., Wang, J.Q., and Zhang, H.Y. "Simplified neutrosophic linguistic multi-criteria group decision-making approach to green product development", Group Decis. Negot., 26(3), pp. 597-627 (2017).

59. Wang, J.Q., Han, Z.Q., and Zhang, H.Y. "Multicriteria group decision-making method based on intuitionistic interval fuzzy information", Group Decis. Negot., pp. 1-19 (2014).

60. Sun, R.X., Hu, J.H., Zhou, J.D., and Chen, X.H. "A hesitant fuzzy linguistic projection-based MABAC method for patients' prioritization", International Journal of Fuzzy Systems (2017). DOI: 10.1007/s40815-017-0345-7

61. Yang, Y., Hu, J.H., An, Q.X., and Chen, X.H. "Group decision making with multiplicative triangular hesitant fuzzy preference relations and cooperative games method ", Int. J. Uncertain. Quantif., 7(3), pp. 271-284 (2017). DOI: 10.1615/Int.J.UncertaintyQuantification. 2017020126 . 


\section{Appendix A}

Theorem 3.1 gives properties of score function, subscore function and sub-accuracy function of SVNE, and the proof of Theorem 3.1 is stated as follows.

\section{Proof of Theorem 3.1}

If $S(\tilde{a})$ be the score function of the SVNE $\tilde{a}$, then $S(\tilde{a})=\left(T_{a}(x)-F_{a}(x)\right)\left(1-I_{a}(x)\right)$; as per Definition 2.2 , we can get $-1 \leq T_{a}(x)-F_{a}(x) \leq 1$ and $0 \leq 1-I_{a}(x) \leq 1$, so the conclusions $-1 \leq S^{\prime}(\tilde{a}) \leq 1$ and $-1 \leq S(\tilde{a}) \leq 1$ are proved. Similarly, the conclusion $0 \leq H^{\prime}(\tilde{a}) \leq 1$ can be proved based on Definition 2.2.

\section{Appendix B}

Theorem 3.2 defines the additive consistency of NFPR, and the proof of Theorem 3.2 is stated as follows.

\section{Proof of Theorem 3.2}

Let $\tilde{A}=\left(\tilde{a}_{i j}\right)_{n \times n}$ be an additive NFPR, then according to Eq. (6), we have:

$$
\begin{aligned}
T_{i j} & \left(1-I_{i j}\right)+T_{j k}\left(1-I_{j k}\right)+T_{k i}\left(1-I_{k i}\right) \\
\quad & =T_{k j}\left(1-I_{k j}\right)+T_{j i}\left(1-I_{j i}\right)+T_{i k}\left(1-I_{i k}\right),
\end{aligned}
$$

for any $i, j, k \in\{1,2, \ldots, n\}$

Then, we can get:

$$
\begin{aligned}
T_{i j}\left(1-I_{i j}\right) & -F_{i j}\left(1-I_{i j}\right)=T_{i k}\left(1-I_{i k}\right) \\
& -F_{i k}\left(1-I_{i k}\right)-\left(T_{j k}\left(1-I_{j k}\right)\right. \\
& \left.-F_{j k}\left(1-I_{j k}\right)\right),
\end{aligned}
$$

based on Eq. (5), so:

$$
\begin{aligned}
\left(T_{i j}-F_{i j}\right)\left(1-I_{i j}\right) & =\left(T_{i k}-F_{i k}\right)\left(1-I_{i k}\right) \\
& -\left(T_{j k}-F_{j k}\right)\left(1-I_{j k}\right) .
\end{aligned}
$$

Therefore, we can obtain $S\left(\tilde{a}_{i j}\right)=S\left(\tilde{a}_{i k}\right)-S\left(\tilde{a}_{j k}\right)$. On the other hand, if $S\left(\tilde{a}_{i j}\right)=S\left(\tilde{a}_{i k}\right)-S\left(\tilde{a}_{j k}\right)$ for any $i, j, k \in\{1,2, \ldots n\}$, then:

$$
\begin{aligned}
T_{i j}\left(1-I_{i j}\right)+ & T_{j k}\left(1-I_{j k}\right)+T_{k i}\left(1-I_{k i}\right) \\
& =T_{k j}\left(1-I_{k j}\right)+T_{j i}\left(1-I_{j i}\right) \\
& +T_{i k}\left(1-I_{i k}\right),
\end{aligned}
$$

for any $i, j, k \in\{1,2, \ldots n\}$, so the $\operatorname{NFPR} \tilde{A}=\left(\tilde{a}_{i j}\right)_{n \times n}$ is additive consistent.

\section{Appendix C}

Theorem 3.3 defines MAD between two NFPRs, and the proof of Theorem 3.3 is stated as follows.

\section{Proof of Theorem 3.3}

1. Because:

$$
\left|T_{a i j}\left(1-I_{a i j}\right)-T_{b i j}\left(1-I_{b i j}\right)\right| \geq 0,
$$

and:

$$
\left|F_{a i j}\left(1-I_{a i j}\right)-F_{b i j}\left(1-I_{b i j}\right)\right| \geq 0,
$$

one can get $\operatorname{MAD}(\tilde{A}, \tilde{B}) \geq 0$; as per Definition 2.2, $T_{a i j}\left(1-I_{a i j}\right), T_{b i j}\left(1-I_{b i j}\right), \quad F_{a i j}\left(1-I_{a i j}\right)$, and $F_{b i j}\left(1-I_{b i j}\right)$ all belong to the interval $[0,1]$ for any $i, j \in\{1,2, \ldots n\}$. Then:

$$
-1 \leq T_{a i j}\left(1-I_{a i j}\right)-T_{b i j}\left(1-I_{b i j}\right) \leq 1
$$

and:

$$
-1 \leq F_{a i j}\left(1-I_{a i j}\right)-F_{b i j}\left(1-I_{b i j}\right) \leq 1 .
$$

It is concluded that:

$$
\left|T_{a i j}\left(1-I_{a i j}\right)-T_{b i j}\left(1-I_{b i j}\right)\right| \leq 1,
$$

and:

$$
\left|F_{a i j}\left(1-I_{a i j}\right)-F_{b i j}\left(1-I_{b i j}\right)\right| \leq 1
$$

so:

$$
\begin{aligned}
& \left|T_{a i j}\left(1-I_{a i j}\right)-T_{b i j}\left(1-I_{b i j}\right)\right|+\mid F_{a i j}\left(1-I_{a i j}\right) \\
& -F_{b i j}\left(1-I_{b i j}\right) \mid \leq 2
\end{aligned}
$$

as per Eq. (13); therefore, $\operatorname{MAD}(\tilde{A}, \tilde{B}) \leq 1$ is proved.

2. As per Eq. (13), since:

$$
\begin{aligned}
& \left|T_{a i j}\left(1-I_{a i j}\right)-T_{b i j}\left(1-I_{b i j}\right)\right| \\
& \quad=\left|T_{b i j}\left(1-I_{b i j}\right)-T_{a i j}\left(1-I_{a i j}\right)\right|,
\end{aligned}
$$

and:

$$
\left|F_{a i j}\left(1-I_{a i j}\right)-F_{b i j}\left(1-I_{b i j}\right)\right|=\mid F_{b i j}\left(1-I_{b i j}\right)
$$

$$
-F_{a i j}\left(1-I_{a i j}\right) \mid,
$$

$\operatorname{MAD}(\tilde{A}, \tilde{B})=\operatorname{MAD}(\tilde{B}, \tilde{A})$ is proved.

3. As $F_{a i j}=T_{a j i}$ in $\tilde{A}$ and $F_{b i j}=T_{b j i}$ in $\tilde{B}$, one can obtain:

$$
\left|F_{a i j}\left(1-I_{a i j}\right)-F_{b i j}\left(1-I_{b i j}\right)\right|=\mid T_{a j i}\left(1-I_{a j i}\right)
$$$$
-T_{b j i}\left(1-I_{b j i}\right) \mid
$$

Since $i, j \in\{1,2, \ldots n\}$ :

$$
\begin{aligned}
& \left|T_{a j i}\left(1-I_{a j i}\right)-T_{b j i}\left(1-I_{b j i}\right)\right|=\mid T_{a i j}\left(1-I_{a i j}\right) \\
& \quad-T_{a i j}\left(1-I_{a i j}\right) \mid .
\end{aligned}
$$

Thus, we can get the conclusion that:

$$
\begin{array}{r}
M A D(\tilde{A}, \tilde{B})=\frac{1}{n(n-1)} \sum_{i=1}^{n} \sum_{j=1, j \neq i}^{n} \\
\left|T_{a i j}\left(1-I_{a i j}\right)-T_{b i j}\left(1-I_{b i j}\right)\right| .
\end{array}
$$




\section{Appendix D}

Definition 4.1 defines additive consistent NFPR, and the proof of Definition 4.1 is stated as follows.

\section{Proof of Definition 4.1}

The NFPR $\hat{A}=\left(\hat{a}_{i j}\right)_{n \times n}$ is additive consistent, because it satisfies the following properties:

(a) $0 \leq \hat{T}_{i j}, \hat{I}_{i j}, \hat{F}_{i j} \leq 1$, for any $i, j \in\{1,2, \ldots, n\}$;

(b) $\hat{T}_{i i}=\hat{F}_{i i}=0.5$ and $\hat{I}_{i i}=0$, for any $i, j \in$ $\{1,2, \ldots n\}$

(c) $\hat{T}_{i j}=\hat{F}_{j i}, \hat{F}_{i j}=\hat{T}_{j i}$, for any $i, j \in\{1,2, \ldots n\}$;

(d) $\hat{T}_{i j}\left(1-\hat{I}_{i j}\right)+\hat{T}_{j k}\left(1-\hat{I}_{j k}\right)+\hat{T}_{k i}\left(1-\hat{I}_{k i}\right)=$ $\hat{T}_{k j}\left(1-\hat{I}_{k j}\right)+\hat{T}_{j i}\left(1-\hat{I}_{j i}\right)+\hat{T}_{i k}\left(1-\hat{I}_{i k}\right)$, for any $i, j \in\{1,2, \ldots n\}$.

The properties (a), (b), and (c) can be easily obtained from Eqs. (5) and (15). As per Eq. (15), we can get:

$$
\begin{aligned}
& \hat{T}_{i j}\left(1-\hat{I}_{i j}\right)+\hat{T}_{j k}\left(1-\hat{I}_{j k}\right)+\hat{T}_{k i}\left(1-\hat{I}_{k i}\right) \\
& \quad=0.5\left(T_{i j} I_{i j}+T_{j i} I_{j i}\right)+0.5\left(T_{j k} I_{j k}+T_{k j} I_{k j}\right) \\
& \quad+0.5\left(T_{k i} I_{k i}+T_{i k} I_{i k}\right)=0.5\left(T_{k j} I_{k j}+T_{j k} I_{j k}\right) \\
& \quad+0.5\left(T_{j i} I_{j i}+T_{i j} I_{i j}\right)+0.5\left(T_{i k} I_{i k}+T_{k i} I_{k i}\right) \\
& \quad=\hat{T}_{k j}\left(1-\hat{I}_{k j}\right)+\hat{T}_{j i}\left(1-\hat{I}_{j i}\right)+\hat{T}_{i k}\left(1-\hat{I}_{i k}\right) .
\end{aligned}
$$

So property (d) is also proved. The proof is completed.

\section{Appendix E}

Theorem 4.1 stated that derived matrix is an additive consistent NFPR, and the proof of Theorem 4.1 is stated as follows.

\section{Proof of Theorem 4.1}

Similar to the proof of Definition 4.1, Theorem 4.1 can be proved as follows.

(a) $0 \leq \tilde{T}_{i j}^{\prime}, \tilde{I}_{i j}^{\prime}, \tilde{F}_{i j}^{\prime} \leq 1$, for any $i, j \in\{1,2, \ldots, n\}$;

(b) $\tilde{T}_{i i}^{\prime}=\tilde{F}_{i i}^{\prime}=0.5$ and $\hat{I}_{i i}=0$, for any $i, j \in$ $\{1,2, \ldots n\}$

(c) $\tilde{T}_{i j}^{\prime}=\tilde{F}_{j i}^{\prime}, \tilde{F}_{i j}^{\prime}=\tilde{T}_{j i}^{\prime}$, for any $i, j \in\{1,2, \ldots n\}$;

(d) $\tilde{T}_{i j}^{\prime}\left(1-\tilde{I}_{i j}^{\prime}\right)+\tilde{T}_{j k}^{\prime}\left(1-\tilde{I}_{j k}^{\prime}\right)+\tilde{T}_{k i}^{\prime}\left(1-\tilde{I}_{k i}^{\prime}\right)=$ $\tilde{T}_{k j}^{\prime}\left(1-\tilde{I}_{k j}^{\prime}\right)+\tilde{T}_{j i}^{\prime}\left(1-\tilde{I}_{j i}^{\prime}\right)+\tilde{T}_{i k}^{\prime}\left(1-\tilde{I}_{i k}^{\prime}\right)$, for any $i, j \in\{1,2, \ldots n\}$.

The proofs of (a)-(c) can be easily obtained based on Eqs. (5) and (16). As per Eq. (16), one can get:

$$
\tilde{T}_{i j}^{\prime}\left(1-\tilde{I}_{i j}^{\prime}\right)+\tilde{T}_{j k}^{\prime}\left(1-\tilde{I}_{j k}^{\prime}\right)+\tilde{T}_{k i}^{\prime}\left(1-\tilde{I}_{k i}^{\prime}\right)
$$

$$
\begin{aligned}
= & \frac{\hat{T}_{i j}\left(1-\hat{I}_{i j}\right)+t}{1+2 t}+\frac{\hat{T}_{j k}\left(1-\hat{I}_{j k}\right)+t}{1+2 t} \\
& +\frac{\hat{T}_{k i}\left(1-\hat{I}_{k i}\right)+t}{1+2 t} \\
& =\frac{\hat{T}_{i j}\left(1-\hat{I}_{i j}\right)+\hat{T}_{j k}\left(1-\hat{I}_{j k}\right)+\hat{T}_{k i}\left(1-\hat{I}_{k i}\right)+3 t}{1+2 t}, \\
\tilde{T}_{k j}^{\prime}\left(1-\tilde{I}_{k j}^{\prime}\right)+\tilde{T}_{j i}^{\prime}\left(1-\tilde{I}_{j i}^{\prime}\right)+\tilde{T}_{i k}^{\prime}\left(1-\tilde{I}_{i k}^{\prime}\right) & \\
= & \frac{\hat{T}_{k j}\left(1-\hat{I}_{k j}\right)+t}{1+2 t}+\frac{\hat{T}_{j i}\left(1-\hat{I}_{j i}\right)+t}{1+2 t} \\
+ & \frac{\hat{T}_{i k}\left(1-\hat{I}_{i k}\right)+t}{1+2 t} \\
= & \frac{\hat{T}_{k j}\left(1-\hat{I}_{k j}\right)+\hat{T}_{j i}\left(1-\hat{I}_{j i}\right)+\hat{T}_{i k}\left(1-\hat{I}_{i k}\right)+3 t}{1+2 t} .
\end{aligned}
$$

According to Eq. (15), proof (d) is proved and the proof of Theorem 4.1 is completed.

\section{Appendix F}

Theorem 4.2 stated that derived matrix is equal to the additive consistent NFPR, and the proof of Theorem 4.2 is stated as follows.

\section{Proof of Theorem 4.2}

Let $\hat{a}_{i j}=\left\langle\hat{T}_{i j}, \hat{I}_{i j}, \hat{F}_{i j}\right\rangle$, then:

$$
\begin{gathered}
\sum_{l=1}^{n}\left(T_{i l}\left(1-I_{i l}\right)-T_{j l}\left(1-I_{j l}\right)\right)-\sum_{l=1}^{n}\left(T_{l i}\left(1-I_{l i}\right)\right. \\
\left.\quad-T_{l j}\left(1-I_{l j}\right)\right)=\sum_{l=1}^{n}\left(T_{i l}\left(1-I_{i l}\right)+T_{l j}\left(1-I_{l j}\right)\right. \\
\left.-T_{j l}\left(1-I_{j l}\right)-T_{l i}\left(1-I_{l i}\right)\right)=\sum_{l=1}^{n}\left(T_{i j}\left(1-I_{i j}\right)\right. \\
\left.-T_{j i}\left(1-I_{j i}\right)\right)=n\left(T_{i j}\left(1-I_{i j}\right)-T_{j i}\left(1-I_{j i}\right)\right) .
\end{gathered}
$$

As per Definition 4.1:

$$
\begin{aligned}
\hat{T}_{i j}\left(1-\hat{I}_{i j}\right)= & 0.5\left(T_{i j}\left(1-I_{i j}\right)-T_{j i}\left(1-I_{j i}\right)\right) \\
& +0.5\left(T_{i j}\left(1-I_{i j}\right)+T_{j i}\left(1-I_{j i}\right)\right),
\end{aligned}
$$

then:

$$
\hat{T}_{i j}\left(1-\hat{I}_{i j}\right)=T_{i j}\left(1-I_{i j}\right),
$$

thus, $\tilde{A}=\tilde{A}^{\prime}$. 


\section{Appendix G}

Theorem 4.3 stated that the weighted averaging matrix obtained from an original NFPR is still an NFPR, and the proof of Theorem 4.3 is stated as follows.

\section{Proof of Theorem 4.3}

As for Definition 4.2 and Eq. (17), one can easily obtain $T_{i j}(\lambda)=F_{j i}(\lambda), F_{i j}(\lambda)=T_{j i}(\lambda), I_{i j}(\lambda)=I_{j i}(\lambda)$, $T_{i i}(\lambda)=F_{i i}(\lambda)=0.5$, and $I_{i i}(\lambda)=0$ for any $i, j \in$ $\{1,2, \ldots n\}$; thus, the proof is completed.

\section{Appendix $\mathbf{H}$}

Theorem 4.4 defined the acceptably consistent NFPR, and the proof of Theorem 4.4 is stated as follows.

\section{Proof of Theorem 4.4}

According to Eq. (10), one can obtain $C I(\tilde{A})=1-$ $\frac{1}{3 n(n-1)(n-2)} \dot{T}(\tilde{A})$, where:

$$
\begin{aligned}
& \dot{T}(\tilde{A})=\sum_{i=1}^{n} \sum_{j=1, j \neq i}^{n} \sum_{k=1, k \neq i, j}^{n} \mid T_{i j}\left(1-I_{i j}\right) \\
& \quad+T_{j k}\left(1-I_{j k}\right)+T_{k i}\left(1-I_{k i}\right)-T_{j i}\left(1-I_{j i}\right) \\
& \quad-T_{k j}\left(1-I_{k j}\right)-T_{i k}\left(1-I_{i k}\right) \mid .
\end{aligned}
$$

Similarly, we can get:

$$
C I(\tilde{A}(\lambda))=1-\frac{1}{3 n(n-1)(n-2)} \dot{T}(\tilde{A}(\lambda)),
$$

where:

$$
\begin{aligned}
& \dot{T}(\tilde{A}(\lambda))=\sum_{i=1}^{n} \sum_{j=1, j \neq i}^{n} \sum_{k=1, k \neq i, j}^{n} \\
& \mid T_{i j}(\lambda)\left(1-I_{i j}(\lambda)\right)+T_{j k}(\lambda)\left(1-I_{j k}(\lambda)\right) \\
& +T_{k i}(\lambda)\left(1-I_{k i}(\lambda)\right)-T_{j i}(\lambda)\left(1-I_{j i}(\lambda)\right) \\
& \quad-T_{k j}(\lambda)\left(1-I_{k j}(\lambda)\right)-T_{i k}(\lambda)\left(1-I_{i k}(\lambda)\right) \mid
\end{aligned}
$$

which means that:

$$
C I(\tilde{A}(\lambda))=1-\frac{1-\lambda}{3 n(n-1)(n-2)} \dot{T}(\tilde{A}),
$$

then:

$$
C I(\tilde{R}(\lambda))=C I(\tilde{R})+\lambda(1-C I(\tilde{R})) .
$$

Therefore:

$$
C I(\tilde{A}(\lambda)) \geq \theta \quad \text { if } \quad \frac{\theta-C I(\tilde{A})}{1-C I(\tilde{A})} \leq \lambda \leq 1 .
$$

Thus, the proof of Theorem 4.4 is completed.

\section{Biographies}

Yan Yang is a PhD degree candidate in Management Science and Engineering in the School of Business, Central South University, China. Her primary research interests lie in decision-making theory and its application in medical big data analysis.

Junhua $\mathbf{H u}$ is a Professor in the School of Business, Central South University, Changsha, China. He received his $\mathrm{PhD}$ degree in Management Engineering from Chiba Institute of Technology, Chiba-ken, Japan. His research interests are in the areas of medical big data analysis, decision-making theory and method, ecommerce, logistics and supply chain management, and financial risk management.

Ruixiao Sun is an MS degree candidate in Management Science and Engineering in the School of Business, Central South University, China. Her current research interests include decision-making theory and its application in medical decision, recommendation system, etc.

Xiaohong Chen is a Professor in the School of Business, Central South University, Changsha, China. She holds the PhD degree from Tokyo Institute of Technology, Tokyo, Japan. Her research interests cover decision theory and decision support system, SMEs financing and management, two-oriented society, etc. 